\title{
Weakly correlated synapses promote dimension reduction in deep neural networks
}

\author{
Jianwen Zhou $\odot$ and Haiping Huang ${ }^{*} *$ \\ PMI Lab, School of Physics, Sun Yat-sen University, Guangzhou 510275, People's Republic of China
}

(Received 24 June 2020; revised 22 December 2020; accepted 8 January 2021; published 29 January 2021)

\begin{abstract}
By controlling synaptic and neural correlations, deep learning has achieved empirical successes in improving classification performances. How synaptic correlations affect neural correlations to produce disentangled hidden representations remains elusive. Here we propose a simplified model of dimension reduction, taking into account pairwise correlations among synapses, to reveal the mechanism underlying how the synaptic correlations affect dimension reduction. Our theory determines the scaling of synaptic correlations requiring only mathematical self-consistency for both binary and continuous synapses. The theory also predicts that weakly correlated synapses encourage dimension reduction compared to their orthogonal counterparts. In addition, these synapses attenuate the decorrelation process along the network depth. These two computational roles are explained by a proposed mean-field equation. The theoretical predictions are in excellent agreement with numerical simulations, and the key features are also captured by deep learning with Hebbian rules.
\end{abstract}

DOI: 10.1103/PhysRevE.103.012315

\section{INTRODUCTION}

Neural correlation is a common characteristic in most neural computations [1], playing vital roles in stimulus coding [2,3], information storage [4], and various cognition tasks that can be implemented by recurrent neural networks $[5,6]$. Using mean-field theory, neural correlation was recently shown to be able to manipulate the dimensionality of layered representations in deep computations [7], which is a fundamental process in deep artificial neural networks [8]. This theory demonstrates that a compact neural representation with weak neural correlations is an emergent behavior of layered neural networks whose synaptic weights are independently and identically distributed. However, in real cortical circuits, synaptic weights among neurons, even in the same layer, may not be ideally independent from each other [4,9-11], perhaps mainly due to biological synaptic plasticity $[4,11]$. On the other hand, a recent theoretical study of unsupervised feature learning predicts that weakly correlated synapses promote unsupervised concept-formation by reducing the necessary amount of sensory data samples $[12,13]$. Therefore, in what exact way a weak correlation among synapses affects the emergent behavior of layered neural networks remains unknown.

In particular, suppression of unwanted variability in sensory inputs [14], compact representation of invariance (generalization to novel context) [15], and feature selectivity (discrimination ability of neural representation) [16] may be closely related to an appropriate level of neural

\footnotetext{
*huanghp7@mail.sysu.edu.cn
}

Published by the American Physical Society under the terms of the Creative Commons Attribution 4.0 International license. Further distribution of this work must maintain attribution to the author(s) and the published article's title, journal citation, and DOI. dimensionality, which keeps only relevant informative components spanning a robust neural subspace [6]. Neural activity in vivo was observed to be constrained on a low-dimensional manifold [17-21]. Therefore, clarifying how synaptic correlations affect neural correlations and further the neural process underlying the transformation of representation dimensionality becomes fundamentally important [22].

To reveal the mechanism underlying how the synaptic correlations affect compact neural representations, we consider deep neural networks that realize a layerwise transformation of sensory inputs. All in-coming synapses to a hidden neuron form the receptive field (RF) of that hidden neuron. The correlation among synapses is modeled by the inter-RF correlation (Fig. 1), in line with our recent work [13]. We do not need a prior knowledge about the synaptic correlation strength. In fact, our mean-field theory yields different scaling behaviors of synaptic correlation with respect to the number of neurons in each layer, for both binary and continuous synaptic weights.

Compared to the previous work on orthogonal weights [7], our current model reveals richer ways of controlling dimensionality of neural representations, i.e., the dimensionality can be tuned layer by layer in both additive and multiplicative manners. Each manner can be analytically understood in the mean-field limit. The theory predicts that the weak correlation among inter-RF synapses is able to promote dimension reduction in deep neural networks. In addition, these synapses attenuate the neural decorrelation process along the network hierarchy. Therefore, our theory provides insights toward understanding how synaptic correlations shape compact neural representations in deep neural networks.

\section{MODEL}

A deep neural network is composed of multiple layers of nonlinear transformations of sensory inputs (e.g., natural images). The depth of the network is defined as the number 


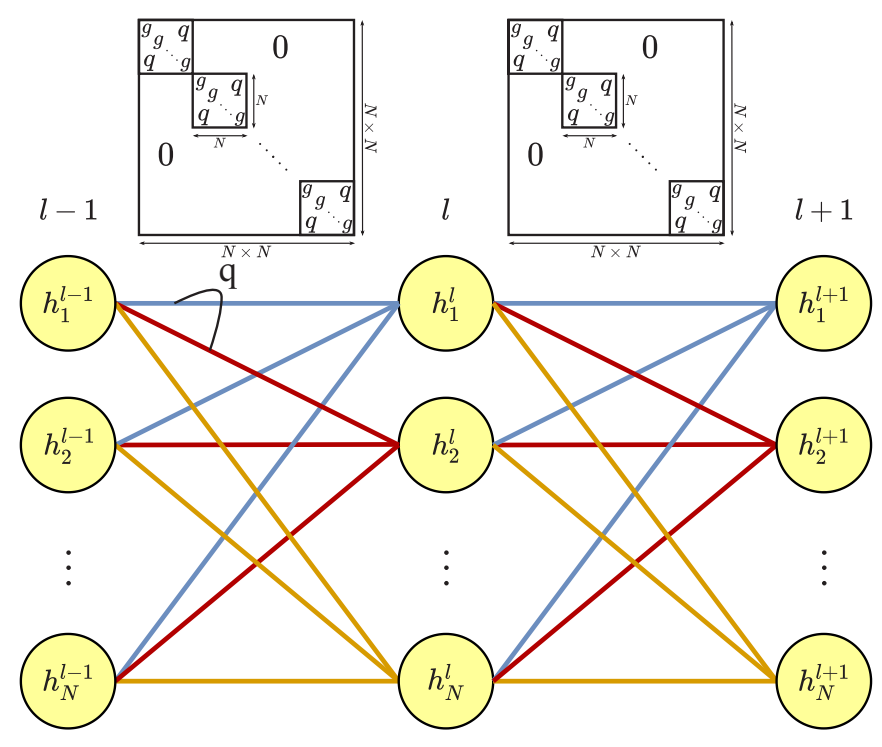

FIG. 1. Schematic illustration of a deep neural network with correlated synapses. During the layerwise transformation of a sensory input, a cascade of internal representations $\left(\left\{\mathbf{h}^{l}\right\}\right)$ are generated by the correlated synapses, with the covariance structure specified by the matrix above the layer. $g$ characterizes the variance of synaptic weights, while the diagonal block (except for the diagonal within the block) characterizes the inter-receptive-field correlation among corresponding synapses (different line colors), and $q$ specifies the synaptic correlation strength. We do not know a priori the exact scaling of $q$, which is self-consistently determined by our theory.

of hidden layers $(d)$, and the width of each layer is defined by the number of neurons at that layer. For simplicity, we assume an equal width $(N)$ in this study. To specify weights between $(l-1)$-th and $l$ th layers, we define a weight matrix $\mathbf{w}^{l}$ whose $i$ th row corresponds to incoming connections to the neuron $i$ at the $l$ th layer (the so-called receptive field of the neuron $i$ ). Firing biases of neurons at the $l$ th layer are denoted by $\mathbf{b}^{l}$. The input data are transformed through consecutive hidden representations denoted by $\mathbf{h}^{l}(l=1, \ldots, d)$, in which each entry $h_{i}^{l}$ defines a nonlinear transformation of its preactivation $z_{i}^{l} \equiv\left[\mathbf{w}^{l} \mathbf{h}^{l-1}\right]_{i}+b_{i}^{l}$, as $h_{i}^{l}=\phi\left(z_{i}^{l}\right)$. For simplicity, we use the nonlinear transfer function $\phi(x)=\tanh (x)$ (note that our theory also applies to other activation functions).

To take into account inter-RF correlations, we specify the covariance structure as $\overline{w_{i k}^{l} w_{j k}^{l}}=q$ for $i \neq j$, and $\overline{\left(w_{i k}^{l}\right)^{2}}=$ $g^{2} / N$ for continuous weights, while $\overline{\left(w_{i k}^{l}\right)^{2}}=1$ for binary weights $( \pm 1)$ (see Fig. 1). The weight has zero mean. denotes the disorder average over the network parameter statistics. The bias follows $\mathcal{N}\left(0, \sigma_{b}\right)$. The orthogonal RF case was studied in a recent work [7] that demonstrates mechanisms of dimension reduction in deep neural networks of continuous weights. Here, we extend the analysis to the nonorthogonal case, and do not enforce any scaling constraint a priori to the correlation level $q$. The role of $q$ in dimension reduction is determined in a self-consistent way. Note that we do not assume any prescribed correlations of intra-RF weights for simplicity (i.e., $\overline{w_{i k}^{l} w_{i s}^{l}}=0$ for $k \neq s$ ). In the current setting, the weight matrix $\mathbf{w}^{l}$ is more highly structured than that in the orthogonal case, resembling qualitatively what occurs in a biological neural circuit $[10,11]$.

To define the computational task, we consider a random input ensemble from which each input sample is drawn. This ensemble is characterized by zero mean and the covariance matrix $\Lambda=\frac{1}{N} \xi \xi^{T}$, where $\xi$ is an $N \times P$ matrix whose components follow a normal distribution of zero mean and variance $\sigma^{2}$ ( $\sigma=0.5$ throughout the paper). The ratio $\alpha=P / N$ controls the spectral density of the covariance matrix (see Appendix A). We remark that this model setting is a simplification of a practical complex learning process, preserving structured weights and correlated input pixels. The simplification allows for the following mean-field analysis, which provides a first step toward understanding rich dimension-tuning behaviors [e.g., dimension compression and expansion during unsupervised learning, or dimension correlates of supervised learning performances (see Appendix B)], and moreover provides hints on how to initialize training well [23-25].

\section{THEORETICAL ANALYSIS IN THE LARGE- $N$ LIMIT}

In this paper, we consider deep neural networks of either binary or continuous weights. In the case of binary weights, the distribution of $w_{i j}^{l}$ is parametrized by zero mean and the covariance given by

$$
\overline{w_{i j}^{l} w_{k s}^{l}}=\delta_{j s} q+\delta_{i k} \delta_{j s}(1-q)=q \delta_{j s}\left(1-\delta_{i k}\right)+\delta_{i k} \delta_{j s},
$$

where $\delta_{j s}$ is the Kronecker delta function. An illustration of this weight covariance is already given in Fig. 1. For simplicity, we consider only inter-RF correlations, neglecting intra-RF ones. The bias parameter $b_{i}^{l}$ independently follows a Gaussian distribution with zero mean and variance $\sigma_{b}$. The activation $h_{i}^{l}$ is then given by

$$
h_{i}^{l}=\phi\left(\frac{g}{\sqrt{N}} \sum_{j} w_{i j}^{l} h_{j}^{l-1}+b_{i}^{l}\right) .
$$

For the continuous-weight case, the parameter $w_{i j}^{l}$ follows a Gaussian distribution with zero mean and the covariance specified by

$$
\overline{w_{i j}^{l} w_{k s}^{l}}=\delta_{j s} q+\delta_{i k} \delta_{j s}\left(\frac{g^{2}}{N}-q\right)=q \delta_{j s}\left(1-\delta_{i k}\right)+\frac{g^{2}}{N} \delta_{i k} \delta_{j s} .
$$

The bias parameter $b_{i}^{l}$ independently follows a Gaussian distribution with zero mean and variance $\sigma_{b}$. The activation $h_{i}^{l}$ is then given by

$$
h_{i}^{l}=\phi\left(\sum_{j} w_{i j}^{l} h_{j}^{l-1}+b_{i}^{l}\right)
$$

\section{A. Mean-field iteration of activity moments}

In this section, we derive the mean-field iteration of activity moments. Note that, for both binary and continuous weights, the form of the iteration looks very similar, we here thus derive in detail the iteration for the continuous case. We first derive 
the mean-field equation for the mean activity $m_{i}^{l}$ as follows:

$$
\begin{aligned}
m_{i}^{l} & =\left\langle h_{i}^{l}\right\rangle \\
& =\left\langle\phi\left(\left[\mathbf{w}^{l} \mathbf{h}^{l-1}\right]_{i}+b_{i}^{l}\right)\right\rangle \\
& =\left\langle\phi\left(a_{i}^{l}+\left[\mathbf{w}^{l} \mathbf{m}^{l-1}\right]_{i}+b_{i}^{l}\right)\right\rangle,
\end{aligned}
$$

where the average $\langle\cdot\rangle$ is defined over the activity statistics throughout the paper, and we define the mean-subtracted weighted-sum (or preactivation) $a_{i}^{l}=\sum_{j} w_{i j}^{l}\left(h_{j}^{l-1}-\left\langle h_{j}^{l-1}\right\rangle\right.$ ), then its expectation is zero, and variance is given by $\Delta_{i j}^{l}=$ $\left\langle a_{i}^{l} a_{j}^{l}\right\rangle=\left[\mathbf{w}^{l} \mathbf{C}^{l-1}\left(\mathbf{w}^{l}\right)^{T}\right]_{i j}$, where $\mathbf{C}$ denotes the covariance matrix of the neural activity. Because $a_{i}^{l}$ is the sum of $N$ nearly independent random terms, as $N \rightarrow \infty$, we apply the central limit theorem, and obtain

$$
m_{i}^{l}=\int D t \phi\left(\sqrt{\Delta_{i i}^{l}} t+\sum_{j} w_{i j}^{l} m_{j}^{l-1}+b_{i}^{l}\right),
$$

where $D t=e^{-t^{2} / 2} d t / \sqrt{2 \pi}$. Then, we consider the covariance of activities. Note that the Gaussian random variable $a_{i}^{l}$ has a variance $\Delta_{i i}^{l}$. The activity covariance is then given by

$$
\begin{aligned}
C_{i j}^{l} & =\left\langle h_{i}^{l} h_{j}^{l}\right\rangle-\left\langle h_{i}^{l}\right\rangle\left\langle h_{j}^{l}\right\rangle \\
& =\left\langle\phi\left(a_{i}^{l}+\left[\mathbf{w}^{l} \mathbf{m}^{l-1}\right]_{i}+b_{i}^{l}\right) \phi\left(a_{j}^{l}+\left[\mathbf{w}^{l} \mathbf{m}^{l-1}\right]_{j}+b_{j}^{l}\right)\right\rangle-m_{i}^{l} m_{j}^{l} \\
& =\int \operatorname{DxDy\phi }\left(\sqrt{\Delta_{i i}^{l}} x+b_{i}^{l}+\left[\mathbf{w}^{l} \mathbf{m}^{l-1}\right]_{i}\right) \phi\left[\sqrt{\Delta_{j j}^{l}}\left(\psi x+y \sqrt{1-\psi^{2}}\right)+b_{j}^{l}+\left[\mathbf{w}^{l} \mathbf{m}^{l-1}\right]_{j}\right]-m_{i}^{l} m_{j}^{l},
\end{aligned}
$$

where $D x=e^{-x^{2} / 2} d x / \sqrt{2 \pi}$ and $\psi=\Delta_{i j}^{l} / \sqrt{\Delta_{i i}^{l} \Delta_{j j}^{l}}$. Note that $a_{i}^{l}$ and $a_{j}^{l}$ are correlated Gaussian random variables. $a_{i}^{l}$ and $a_{j}^{l}$ have been parametrized by two independent standard Gaussian random variables, say $x$ and $y$, respectively. The preactivation correlation has been captured by the correlation coefficient $\psi(|\psi| \leqslant 1)$.

For binary weights, the above Eqs. (6) and (7) become

$$
m_{i}^{l}=\int \operatorname{Dt\phi }\left(\sqrt{\Delta_{i i}^{l}} t+\frac{g}{\sqrt{N}} \sum_{j} w_{i j}^{l} m_{j}^{l-1}+b_{i}^{l}\right),
$$

and

$$
C_{i j}^{l}=\int D x D y \phi\left(\sqrt{\Delta_{i i}^{l}} x+b_{i}^{l}+\frac{g}{\sqrt{N}}\left[\mathbf{w}^{l} \mathbf{m}^{l-1}\right]_{i}\right) \phi\left[\sqrt{\Delta_{j j}^{l}}\left(\psi x+y \sqrt{1-\psi^{2}}\right)+b_{j}^{l}+\frac{g}{\sqrt{N}}\left[\mathbf{w}^{l} \mathbf{m}^{l 1}\right]_{j}\right]-m_{i}^{l} m_{j}^{l}
$$

where

$$
\Delta_{i j}^{l}=\frac{g^{2}}{N}\left[\mathbf{w}^{l} \mathbf{C}^{l-1}\left(\mathbf{w}^{l}\right)^{T}\right]_{i j}
$$

With the activity moments, we can then evaluate the dimensionality of the $l$ th layer by

$$
D^{l}=\frac{\left(\sum_{i} \lambda_{i}\right)^{2}}{\sum_{i} \lambda_{i}^{2}}=\frac{\left(\operatorname{Tr} \mathbf{C}^{l}\right)^{2}}{\operatorname{Tr}\left(\mathbf{C}^{l}\right)^{2}}=\frac{\left(\sum_{i} C_{i i}^{l}\right)^{2}}{\sum_{i, j}\left(C_{i j}^{l}\right)^{2}},
$$

where $\left\{\lambda_{i}\right\}$ is the eigenspectrum of the covariance matrix $\mathbf{C}^{l}$. Then we can define the normalized dimensionality as $\tilde{D}^{l}=\frac{\left(\operatorname{Tr} \mathbf{C}^{l}\right)^{2}}{N \operatorname{Tr}\left(\mathbf{C}^{2}\right)^{2}}$, which is then independent of the network width $N$. To derive the recursion of dimensionality for each layer, we define additionally $\mathcal{K}_{1}^{l}=\frac{1}{N} \sum_{i} C_{i i}^{l}, \mathcal{K}_{2}^{l}=\frac{1}{N} \sum_{i}\left(C_{i i}^{l}\right)^{2}$, and $\Sigma^{l}=\frac{2}{N^{2}} \sum_{i<j}\left(C_{i j}^{l}\right)^{2}$ for a large value of $N$. The normalized dimensionality of the $l$ th layer is thus expressed as

$$
\tilde{D}^{l}=\frac{\left(\mathcal{K}_{1}^{l}\right)^{2}}{N \Sigma^{l}+\mathcal{K}_{2}^{l}},
$$

which is useful for the following theoretical analysis.

\section{B. Expansion of two-point correlations}

In the mean-field limit, we can assume $C_{i j}^{l} \sim \mathcal{O}(1 / \sqrt{N})$ for $i \neq j[26]$. This is the clustering property of pure states in physics, requiring that the connected correlation functions decay to zero at large distances. This property could be verified in simulations. In the following part, we assume weights take continuous values. Binary weights can be similarly analyzed, by noting that the preactivations should be multiplied by a prefactor $g / \sqrt{N}$.

We first analyze the off-diagonal part of the covariance matrix. First, we notice that $\overline{\Delta_{i j}^{2}}=\sum_{k, l} \overline{w_{i k}^{2} w_{j l}^{2}} C_{k l}^{2}=N^{2} \frac{g^{2}}{N} \frac{g^{2}}{N} \frac{1}{N} \sim$ $\mathcal{O}\left(\frac{g^{4}}{N}\right)$, which means that $\Delta_{i j} \sim \mathcal{O}\left(\frac{g^{2}}{\sqrt{N}}\right)$. That is, when $N$ is sufficiently large, $\Delta_{i j}$ is very small. Then we can carry out a Taylor expansion with respect to a small $\Delta_{i j}$ whose layer index is added below:

$$
\begin{aligned}
\phi & {\left[\sqrt{\Delta_{j j}^{l}}\left(\psi x+y \sqrt{1-\psi^{2}}\right)+z_{j}^{0}\right] } \\
& =\phi\left(\sqrt{\Delta_{j j}^{l}} y+z_{j}^{0}\right)+\phi^{\prime}\left(\sqrt{\Delta_{j j}^{l}} y+z_{j}^{0}\right) \frac{x \Delta_{i j}^{l}}{\sqrt{\Delta_{i i}^{l}}}+\mathcal{O}\left(\left(\Delta_{i j}^{l}\right)^{2}\right),
\end{aligned}
$$

where we define $z_{j}^{0}=b_{j}^{l}+\left[\mathbf{w}^{l} \mathbf{m}^{l-1}\right]_{j}$. By noting that $m_{i}^{l}=$ $\int \operatorname{Dt} \phi\left(\sqrt{\Delta_{i i}^{l}} t+z_{i}^{0}\right)$, we obtain 


$$
\begin{aligned}
C_{i j}^{l} & =\int \operatorname{DxDy\phi }\left(\sqrt{\Delta_{i i}^{l}} x+z_{i}^{0}\right) \phi^{\prime}\left(\sqrt{\Delta_{j j}^{l}} y+z_{j}^{0}\right) \frac{x \Delta_{i j}^{l}}{\sqrt{\Delta_{i i}^{l}}}+\mathcal{O}\left(\left(\Delta_{i j}^{l}\right)^{2}\right) \\
& =\int \operatorname{DxDy} \phi^{\prime}\left(\sqrt{\Delta_{i i}^{l}} x+z_{i}^{0}\right) \phi^{\prime}\left(\sqrt{\Delta_{j j}^{l}} y+z_{j}^{0}\right) \Delta_{i j}^{l}+\mathcal{O}\left(\left(\Delta_{i j}^{l}\right)^{2}\right) .
\end{aligned}
$$

To derive the second equality, we have used the property of Gaussian integral: $\int D x \phi(x) x=\int D x \phi^{\prime}(x)$. Therefore we can write $C_{i j}^{l} \simeq\left\langle\phi^{\prime}\left(\sqrt{\Delta_{i i}^{l}} x+z_{i}^{0}\right)\right\rangle_{x}\left\langle\phi^{\prime}\left(\sqrt{\Delta_{j j}^{l}} x+z_{j}^{0}\right)\right\rangle_{y} \Delta_{i j}^{l}$, where the linear coefficient is an average over standard normal variables, and is called hereafter $K_{i j}^{l}$ for the following analysis.

We next remark that $\overline{\Delta_{i i}} \simeq \frac{g^{2}}{N} \sum_{k} \overline{w_{i k}^{2}} C_{k k}=g^{2} \mathcal{K}_{1} \sim \mathcal{O}\left(g^{2}\right)$. In the small-g limit, we can carry out an expansion in $\sqrt{\Delta_{i i}}$ whose layer index is added below, and get

$$
\begin{aligned}
C_{i j}^{l} & \simeq \int D x D y\left[\phi^{\prime}\left(z_{i}^{0}\right)+\phi^{\prime \prime}\left(z_{i}^{0}\right) \sqrt{\Delta_{i i}^{l}} x\right]\left[\phi^{\prime}\left(z_{j}^{0}\right)+\phi^{\prime \prime}\left(z_{j}^{0}\right) \sqrt{\Delta_{j j}^{l} y}\right] \Delta_{i j}^{l} \\
& =\phi^{\prime}\left(z_{i}^{0}\right) \phi^{\prime}\left(z_{j}^{0}\right) \Delta_{i j}^{l} .
\end{aligned}
$$

This result was reported in our previous work [7]. We then analyze the diagonal part of the covariance matrix,

$$
\begin{aligned}
C_{i i}^{l} & =\left\langle h_{i}^{l} h_{i}^{l}\right\rangle-\left\langle h_{i}^{l}\right\rangle\left\langle h_{i}^{l}\right\rangle \\
& =\left\langle\phi^{2}\left(a_{i}^{l}+z_{i}^{0}\right)\right\rangle-m_{i}^{l} m_{i}^{l} \\
& =\int D x \phi^{2}\left(\sqrt{\Delta_{i i}^{l}} x+z_{i}^{0}\right)-\int \operatorname{Dx} \phi\left(\sqrt{\Delta_{i i}^{l}} x+z_{i}^{0}\right) \int \operatorname{Dy} \phi\left(\sqrt{\Delta_{i i}^{l}} y+z_{i}^{0}\right) .
\end{aligned}
$$

We expand the above formula in the small $\Delta_{i i}^{l}$, i.e., $\phi\left(a_{i}^{l}+z_{i}^{0}\right)=\phi\left(z_{i}^{0}\right)+\phi^{\prime}\left(z_{i}^{0}\right) \sqrt{\Delta_{i i}^{l}} x$, and obtain

$$
\begin{aligned}
C_{i i}^{l} & \simeq \int D x\left[\phi\left(z_{i}^{0}\right)+\phi^{\prime}\left(z_{i}^{0}\right) \sqrt{\Delta_{i i}^{l}} x\right]^{2}-\left\{\int D x\left[\phi\left(z_{i}^{0}\right)+\phi^{\prime}\left(z_{i}^{0}\right) \sqrt{\Delta_{i i}^{l}} x\right]\right\}^{2} . \\
& =\left[\phi^{\prime}\left(z_{i}^{0}\right)\right]^{2} \Delta_{i i}^{l} .
\end{aligned}
$$

Therefore, we can write $C_{i i}^{l} \simeq K_{i i}^{l} \Delta_{i i}^{l}$, where $K_{i i}^{l}$ is the shorthand for the linear coefficient. To improve the prediction accuracy, one needs to include high-order terms into this approximation. We observe that, if we use Eq. (14) by setting $i=j$, the theoretical prediction can match the numerical simulation results even in a relatively large value of $g$. This may be due to the fact that the contribution of $\Delta_{i i}^{l}$ is taken into account when computing $K_{i i}^{l}$. For binary weights, the expanded covariance is just the same as the equations [Eq. (14) and Eq. (17)], yet with $z_{i}^{0}=b_{i}^{l}+\frac{g}{\sqrt{N}}\left[\mathbf{w}^{l} \mathbf{m}^{l-1}\right]_{i}$.

\section{Iteration of the correlation strength $\Sigma^{l}$}

\section{Binary weights}

First, we calculate $\mathcal{K}_{1}^{l}$, and in the large- $N$ and small-g limits, we obtain

$$
\begin{aligned}
& \mathcal{K}_{1}^{l}=\overline{\left[\phi^{\prime}\left(\frac{g}{\sqrt{N}} \sum_{j=1}^{N} w_{i j}^{l} m_{j}^{l-1}+b_{i}^{l}\right)\right]^{2}} \Delta_{i i}^{l} \\
& \simeq \overline{\left[\phi^{\prime}\left(\frac{g}{\sqrt{N}} \sum_{j=1}^{N} w_{i j}^{l} m_{j}^{l-1}+b_{i}^{l}\right)\right]^{2}} g^{2} \mathcal{K}_{1}^{l-1},
\end{aligned}
$$

where - means an average over the distribution of network parameters, and $\Delta_{i i}^{l}$ is approximated by

$$
\Delta_{i i}^{l} \simeq \frac{g^{2}}{N} \overline{\sum_{k, j}^{N} w_{i k}^{l} w_{i j}^{l} C_{k j}^{l-1}}=\frac{g^{2}}{N} \sum_{k=1}^{N} C_{k k}^{l-1}=g^{2} \mathcal{K}_{1}^{l-1} .
$$

Note that the argument of $\phi^{\prime}(\cdot)$ is a sum of a large number of nearly independent random variables. It is then easy to write that $\frac{g}{\sqrt{N} \sum_{j} w_{i j}^{l} m_{j}^{l-1}+b_{i}^{l}}=0$, and

$$
\overline{\left(\frac{g}{\sqrt{N}} \sum_{j} w_{i j}^{l} m_{j}^{l-1}+b_{i}^{l}\right)^{2}}=\frac{g^{2}}{N} \sum_{j}\left(m_{j}^{l-1}\right)^{2}+\sigma_{b} .
$$

According to the central limit theorem, we obtain

$$
\begin{aligned}
& \overline{\left[\phi^{\prime}\left(\frac{g}{\sqrt{N}} \sum_{j=1}^{N} w_{i j}^{l} m_{j}^{l-1}+b_{i}^{l}\right)\right]^{2}} \\
& =\int D x\left[\phi^{\prime}\left(x \sqrt{g^{2} Q^{l-1}+\sigma_{b}}\right)\right]^{2} \stackrel{\text { def }}{=} \overline{K_{i i}^{l}},
\end{aligned}
$$


where we have defined $Q^{l-1} \stackrel{\text { def }}{=} \frac{1}{N} \sum_{i=1}^{N}\left(m_{i}^{l-1}\right)^{2}$. The recursion of $Q^{l}$ becomes

$$
\begin{aligned}
Q^{l} & =\frac{1}{N} \sum_{i}\left[\int D t \phi\left(\sqrt{\Delta_{i i}^{l}} t+\frac{g}{\sqrt{N}} \sum_{j} w_{i j} m_{j}^{l-1}+b_{i}^{l}\right)\right]^{2} \\
& =\int D x\left[\int D t \phi\left(\sqrt{g^{2} \mathcal{K}_{1}^{l-1}} t+x \sqrt{g^{2} Q^{l-1}+\sigma_{b}}\right)\right]^{2}
\end{aligned}
$$

where we have used $\Delta_{i i}^{l}=g^{2} \mathcal{K}_{1}^{l-1}$.

Finally, we obtain the recursion for $\mathcal{K}_{1}^{l}$,

$$
\mathcal{K}_{1}^{l}=g^{2} \overline{K_{i i}^{l}} \mathcal{K}_{1}^{l-1} .
$$

Note that $\mathcal{K}_{1}^{l}$ can also be calculated recursively without the small- $g$ assumption as follows:

$$
\begin{aligned}
\mathcal{K}_{1}^{l} & =\int \operatorname{DxDt\phi ^{2}}\left(\sqrt{g^{2} \mathcal{K}_{1}^{l-1}} t+x \sqrt{\sigma_{b}+g^{2} Q^{l-1}}\right)-Q^{l} \\
& =\int \operatorname{Dx} \phi^{2}\left(\sqrt{g^{2} \mathcal{K}_{1}^{l-1}+\sigma_{b}+g^{2} Q^{l-1}} x\right)-Q^{l},
\end{aligned}
$$

where the first equality is derived according to the definition of $\mathcal{K}_{1}^{l}$, and the second equality is derived thanks to the fact that the sum of two independent Gaussian random variables is still Gaussian distributed.

Next, the recursion of $\mathcal{K}_{2}^{l}$ can be calculated by definition as follows:

$$
\begin{aligned}
\mathcal{K}_{2}^{l} & =\frac{1}{N} \sum_{i}\left(C_{i i}^{l}\right)^{2} \\
& =\frac{1}{N} \sum_{i}\left(K_{i i}^{l} g^{2} \mathcal{K}_{1}^{l-1}\right)^{2} \\
& =\overline{\left(K_{i i}^{l}\right)^{2}} g^{4}\left(\mathcal{K}_{1}^{l-1}\right)^{2},
\end{aligned}
$$

where we have assumed that $K_{i i}^{l}$ in the large- $N$ limit does not depend on the specific site index, and thus

$$
\begin{aligned}
\overline{\left(K_{i i}^{l}\right)^{2}} & =\overline{\left[\phi^{\prime}\left(\frac{g}{\sqrt{N}} \sum_{j} w_{i j}^{l} m_{j}^{l-1}+b_{i}^{l}\right)\right]^{4}} \\
& =\int D x\left[\phi^{\prime}\left(x \sqrt{g^{2} Q^{l-1}+\sigma_{b}}\right)\right]^{4} .
\end{aligned}
$$

Note that $\mathcal{K}_{2}^{l}$ can be evaluated recursively without the small-g assumption as

$$
\mathcal{K}_{2}^{l}=\left\langle\left[\left\langle\phi^{2}(f)\right\rangle_{z}-\langle\phi(f)\rangle_{z}^{2}\right]^{2}\right\rangle_{u, t},
$$

where $z, u$, and $t$ are all standard normal variables, and $f \stackrel{\text { def }}{=}$

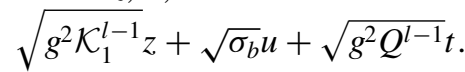

We finally derive the recursion of $\Sigma^{l}$. First, for the binary weights, to compute $\Delta_{i j}^{2}$, where the layer index can be added later, we have

$$
\begin{aligned}
\left(\sum_{k, l} w_{i k} w_{j l} C_{k l}\right)^{2}= & \left(\sum_{k \neq l} w_{i k} w_{j l} C_{k l}+\sum_{k} w_{i k} w_{j k} C_{k k}\right)^{2} \\
\simeq & \sum_{k \neq l ; k^{\prime} \neq l^{\prime}} w_{i k} w_{i k^{\prime}} w_{j l} w_{j l^{\prime}} C_{k l} C_{k^{\prime} l^{\prime}} \\
& +\sum_{k, k^{\prime}} w_{i k} w_{j k} w_{i k^{\prime}} w_{j k^{\prime}} C_{k k} C_{k^{\prime} k^{\prime}} \\
\simeq & \left(1+q^{2}\right) \sum_{k \neq l} C_{k l}^{2}+\left(1-q^{2}\right) \sum_{k} C_{k k}^{2} \\
& +q^{2}\left(\sum_{k} C_{k k}\right)^{2},
\end{aligned}
$$

where the cross-term vanishes in statistics to derive the second equality, due to the vanishing intra-RF correlation for one hidden neuron. The third equality is derived by considering the inter-RF correlation in our current setting. Finally, we arrive at

$$
\begin{aligned}
N \Sigma^{l+1}= & \frac{2}{N} \sum_{i<j} \overline{\left(K_{i j}^{l+1}\right)^{2}} \frac{g^{4}}{N^{2}}\left[\left(1+q^{2}\right) N^{2} \Sigma^{l}\right. \\
& \left.+\left(1-q^{2}\right) N \mathcal{K}_{2}^{l}+q^{2} N^{2}\left(\mathcal{K}_{1}^{l}\right)^{2}\right] \\
= & \overline{\left(K_{i j}^{l+1}\right)^{2}} g^{4}\left[\left(1+q^{2}\right) N \Sigma^{l}+q^{2} N\left(\mathcal{K}_{1}^{l}\right)^{2}+\left(1-q^{2}\right) \mathcal{K}_{2}^{l}\right] \\
\simeq & \overline{\left(K_{i j}^{l+1}\right)^{2}} g^{4}\left[N \Sigma^{l}+\mathcal{K}_{2}^{l}+r^{2}\left(\mathcal{K}_{1}^{l}\right)^{2}\right],
\end{aligned}
$$

where we have to assume $q=r / \sqrt{N}[r \sim \mathcal{O}(1)]$; otherwise Eq. (28) could not hold because of $N \Sigma$ being the order one, or the correlation $q$ plays no role (i.e., identical to the case of $q=0) . \overline{\left(K_{i j}^{l+1}\right)^{2}}$ is used to replace $\left(K_{i j}^{l+1}\right)^{2}$ in the mean-field approximation and can be computed recursively as follows:

$$
\begin{aligned}
\overline{\left(K_{i j}^{l+1}\right)^{2}}= & \left\langle\left\langle\left.\phi^{\prime}\left(\sqrt{g^{2} \mathcal{K}_{1}^{l}} x+\sqrt{g^{2} Q^{l}} z_{1}+\sqrt{\sigma_{b}} u_{1}\right)\right|_{x} ^{2}\right.\right. \\
& \times\left\langle\phi ^ { \prime } \left(\sqrt{g^{2} \mathcal{K}_{1}^{l}} y+\sqrt{g^{2} Q^{l}}\left(\rho z_{1}+\sqrt{1-\rho^{2}} z_{2}\right)\right.\right. \\
& \left.\left.\left.+\sqrt{\sigma_{b}} u_{2}\right)\right\rangle_{y}^{2}\right\rangle_{z_{1}, z_{2}, u_{1}, u_{2}},
\end{aligned}
$$

where $x, y, z_{1}, z_{2}, u_{1}, u_{2}$ are all standard Gaussian random variables, capturing both thermal and disorder average (inner and outer ones, respectively). The correlation coefficient is given by

$$
\rho \stackrel{\text { def }}{=} \frac{\overline{\left(z_{i}^{0}-b_{i}\right)\left(z_{j}^{0}-b_{j}\right)}}{\sqrt{\overline{\left(z_{i}^{0}-b_{i}\right)^{2}\left(z_{j}^{0}-b_{j}\right)^{2}}}}=q .
$$

Therefore, $q$ plays an important role in our current theory, in contrast to the orthogonal case [7]. 


\section{Continuous weights}

First, we calculate $\mathcal{K}_{1}^{l}$, and in the large- $N$ limit, we obtain

$$
\begin{aligned}
& \mathcal{K}_{1}^{l}=\overline{\left[\phi^{\prime}\left(\sum_{j} w_{i j}^{l} m_{j}^{l-1}+b_{i}^{l}\right)\right]^{2}} \Delta_{i i}^{l} \\
& \simeq \overline{\left[\phi^{\prime}\left(\sum_{j} w_{i j}^{l} m_{j}^{l-1}+b_{i}^{l}\right)\right]^{2}} g^{2} \mathcal{K}_{1}^{l-1},
\end{aligned}
$$

where - means an average over the distribution of network parameters and $\Delta_{i i}^{l}$ can be approximated by

$$
\Delta_{i i}^{l} \simeq \overline{\sum_{k, j}^{N} w_{i k}^{l} w_{i j}^{l} C_{k j}^{l-1}}=\frac{g^{2}}{N} \sum_{k=1}^{N} C_{k k}^{l-1}=g^{2} \mathcal{K}_{1}^{l-1}
$$

The argument of $\phi^{\prime}(\cdot)$ is a sum of a large number of nearlyindependent random variables, as a result, it is easy to write that $\overline{\sum_{j} w_{i j}^{l} m_{j}^{l-1}+b_{i}^{l}}=0$, and

$$
\overline{\left(\sum_{j} w_{i j}^{l} m_{j}^{l-1}+b_{i}^{l}\right)^{2}}=\frac{g}{N} \sum_{j}\left(m_{j}^{l-1}\right)^{2}+\sigma_{b} .
$$

According to the central limit theorem, we obtain

$$
\begin{aligned}
\overline{\left[\phi^{\prime}\left(\sum_{j=1}^{N} w_{i j}^{l} m_{j}^{l-1}+b_{i}^{l}\right)\right]^{2}} & =\int D x\left[\phi^{\prime}\left(x \sqrt{g^{2} Q^{l-1}+\sigma_{b}}\right)\right]^{2} \\
& \stackrel{\text { def } \overline{K_{i i}^{l}}}{ }
\end{aligned}
$$

where we have defined $Q^{l-1} \stackrel{\text { def }}{=} \frac{1}{N} \sum_{i}\left(m_{i}^{l-1}\right)^{2}$. The recursion of $Q^{l}$ becomes

$$
\begin{aligned}
Q^{l} & =\frac{1}{N} \sum_{i}\left[\int D t \phi\left(\sqrt{\Delta_{i i}^{l}} t+\sum_{j} w_{i j} m_{j}^{l-1}+b_{i}^{l}\right)\right]^{2} \\
& =\int D x\left[\int D t \phi\left(\sqrt{g^{2} \mathcal{K}_{1}^{l-1}} t+x \sqrt{g^{2} Q^{l-1}+\sigma_{b}}\right)\right]^{2},
\end{aligned}
$$

where we have used $\Delta_{i i}^{l}=g^{2} \mathcal{K}_{1}^{l-1}$.

Finally, we obtain the recursion for $\mathcal{K}_{1}^{l}$ as

$$
\mathcal{K}_{1}^{l}=g^{2} \overline{K_{i i}^{l}} \mathcal{K}_{1}^{l-1}
$$

Note that $\mathcal{K}_{1}^{l}$ can also be calculated recursively without the small- $g$ assumption as follows,

$$
\mathcal{K}_{1}^{l}=\int D x \phi^{2}\left(\sqrt{g^{2} \mathcal{K}_{1}^{l-1}+\sigma_{b}+g^{2} Q^{l-1}} x\right)-Q^{l} .
$$

We then derive $\mathcal{K}_{2}^{l}$ as follows:

$$
\begin{aligned}
\mathcal{K}_{2}^{l} & =\frac{1}{N} \sum_{i}\left(C_{i i}^{l}\right)^{2} \\
& =\frac{1}{N} \sum_{i}\left(K_{i i}^{l} g^{2} \mathcal{K}_{1}^{l-1}\right)^{2} \\
& =\overline{\left(K_{i i}^{l}\right)^{2}} g^{4}\left(\mathcal{K}_{1}^{l-1}\right)^{2},
\end{aligned}
$$

where $\left(K_{i i}^{l}\right)^{2}$ is approximated by

$$
\begin{aligned}
\overline{\left(K_{i i}^{l}\right)^{2}} & =\overline{\left[\phi^{\prime}\left(\sum_{j} w_{i j}^{l} m_{j}^{l-1}+b_{i}^{l}\right)\right]^{4}} \\
& =\int D x\left[\phi^{\prime}\left(x \sqrt{g^{2} Q^{l-1}+\sigma_{b}}\right)\right]^{4}
\end{aligned}
$$

Note that $\mathcal{K}_{2}^{l}$ can be evaluated recursively without the small- $g$ assumption as

$$
\mathcal{K}_{2}^{l}=\left\langle\left[\left\langle\phi^{2}(f)\right\rangle_{z}-\langle\phi(f)\rangle_{z}^{2}\right]^{2}\right\rangle_{u, t}
$$

where $z, u$, and $t$ are all standard normal variables, and $f \stackrel{\text { def }}{=}$ $\sqrt{g^{2} \mathcal{K}_{1}^{l-1}} z+\sqrt{\sigma_{b}} u+\sqrt{g^{2} Q^{l-1}} t$.

To finally obtain the recursion of $\Sigma^{l}$, we first analyze $\Delta_{i j}^{2}$ whose layer index will be added later as follows,

$$
\begin{aligned}
\Delta_{i j}^{2}= & \left(\sum_{k, l} w_{i k} w_{j l} C_{k l}\right)^{2} \\
= & \left(\sum_{k \neq l} w_{i k} w_{j l} C_{k l}+\sum_{k} w_{i k} w_{j k} C_{k k}\right)^{2} \\
\simeq & \sum_{k \neq l ; k^{\prime} \neq l^{\prime}} w_{i k} w_{i k^{\prime}} w_{j l} w_{j l^{\prime}} C_{k l} C_{k^{\prime} l^{\prime}} \\
& +\sum_{k, k^{\prime}} w_{i k} w_{j k} w_{i k^{\prime}} w_{j k^{\prime}} C_{k k} C_{k^{\prime} k^{\prime}} \\
\simeq & \left(\frac{g^{4}}{N^{2}}+q^{2}\right) \sum_{k \neq l} C_{k l}^{2}+\frac{g^{4}}{N^{2}}\left(2 \rho^{2}+1\right) \sum_{k} C_{k k}^{2} \\
& +q^{2}\left[\left(\sum_{k} C_{k k}\right)^{2}-\sum_{k} C_{k k}^{2}\right],
\end{aligned}
$$

where $\rho=\frac{q N}{g^{2}}$ due to the fact that $\overline{w_{i k} w_{j k}}=q$ as well as $\overline{w_{i k}^{2}}=$ $g^{2} / N$. To arrive at the final equality, we have used the statistics equality as follows:

$$
\begin{aligned}
\overline{w_{i k}^{2} w_{j k}^{2}} & =\left\langle\frac{g^{2}}{N} z_{1}^{2} \times \frac{g^{2}}{N}\left(\rho z_{1}+\sqrt{1-\rho^{2}} z_{2}\right)^{2}\right\rangle_{z_{1}, z_{2} \sim \mathcal{N}(0,1)} \\
& =\frac{g^{4}}{N^{2}}\left(2 \rho^{2}+1\right)
\end{aligned}
$$


We finally arrive at the recursion for $\Sigma^{l}$ as follows:

$$
\begin{aligned}
N \Sigma^{l} & \simeq \frac{2}{N} \sum_{i<j} \overline{\left(K_{i j}^{l}\right)^{2}}\left[\left(\frac{g^{4}}{N^{2}}+q^{2}\right) \sum_{k \neq j}\left(C_{k j}^{l-1}\right)^{2}+\left(q^{2}+\frac{g^{4}}{N^{2}}\right) \sum_{k}\left(C_{k k}^{l-1}\right)^{2}+q^{2}\left(\sum_{k} C_{k k}^{l-1}\right)^{2}\right] \\
& =\overline{\left(K_{i j}^{l}\right)^{2}}\left[\left(g^{4}+N^{2} q^{2}\right)\left(N \Sigma^{l-1}+\mathcal{K}_{2}^{l-1}\right)+q^{2} N^{3}\left(\mathcal{K}_{1}^{l-1}\right)^{2}\right] \\
& =\overline{\left(K_{i j}^{l}\right)^{2}}\left[g^{4}\left(N \Sigma^{l-1}+\mathcal{K}_{2}^{l-1}\right)+r^{2}\left(\mathcal{K}_{1}^{l-1}\right)^{2}\right],
\end{aligned}
$$

where we have to assume $q=r / N^{\frac{3}{2}}[r \sim \mathcal{O}(1)]$; otherwise, Eq. (42) could not hold because of $N \Sigma$ being the order one, or the correlation $q$ plays no role (i.e., identical to the case of $q=0$ ). $\overline{\left(K_{i j}^{l}\right)^{2}}$ is used to approximate $\left(K_{i j}^{l}\right)^{2}$ and can be computed as follows:

$$
\begin{aligned}
\overline{\left(K_{i j}^{l}\right)^{2}}= & \left\langle\left\langle\left.\phi^{\prime}\left(\sqrt{g^{2} \mathcal{K}_{1}^{l-1}} x+\sqrt{g^{2} Q^{l-1}} z_{1}+\sqrt{\sigma_{b}} u_{1}\right)\right|_{x} ^{2}\right.\right. \\
& \left.\times\left\langle\phi^{\prime}\left[\sqrt{g^{2} \mathcal{K}_{1}^{l-1}} y+\sqrt{g^{2} Q^{l-1}}\left(\rho z_{1}+\sqrt{1-\rho^{2}} z_{2}\right)+\sqrt{\sigma_{b}} u_{2}\right]\right\rangle_{y}^{2}\right\rangle_{z_{1}, z_{2}, u_{1}, u_{2}},
\end{aligned}
$$

where $x, y, z_{1}, z_{2}, u_{1}, u_{2}$ are all standard Gaussian random variables, capturing both thermal and disorder average (inner and outer ones, respectively). The correlation coefficient in the continuous-weight case is given by

$$
\rho \stackrel{\text { def }}{=} \frac{\overline{\left(z_{i}^{0}-b_{i}\right)\left(z_{j}^{0}-b_{j}\right)}}{\sqrt{\overline{\left(z_{i}^{0}-b_{i}\right)^{2}\left(z_{j}^{0}-b_{j}\right)^{2}}}}=\frac{q N}{g^{2}} .
$$

We finally remark that the synaptic correlation is able to boost the neural correlation level when transmitting signal via hidden representations. From the linear relationship between $\Sigma^{l+1}$ and $\Sigma^{l}$ [see Eq. (28)], one derives for the binary weights that the operating point is given by:

$$
\Sigma_{*}^{l}=\frac{\Upsilon \mathcal{K}_{2}^{l}}{1-\Upsilon}+\frac{\Upsilon r^{2}\left(\mathcal{K}_{1}^{l}\right)^{2}}{1-\Upsilon}
$$

where $\Sigma_{*}^{l}$ has been multiplied by $N$, and $\Upsilon \stackrel{\text { def }}{=} g^{4} \overline{\left(K_{i j}^{l+1}\right)^{2}}$. Eq. (45) implies that the operating point is increased by the synaptic correlations (the last term in the equation). The intercept of the linear relationship is also increased by a positive amount $\Upsilon r^{2}\left(\mathcal{K}_{1}^{l}\right)^{2}$. Note that the slope of the linear relationship under the orthogonal-weight and correlated-weight cases are the same. These effects are qualitatively the same for both continuous and binary weights, which is shown in Fig. 2.

\section{Iteration of the dimensionality across layers \\ 1. Binary weights}

According to the definition, with the help of Eqs. (22) and (24) and the recursion equation for $\Sigma^{l}$, we obtain

$$
\begin{aligned}
\tilde{D}^{l} & =\frac{\left(\mathcal{K}_{1}^{l}\right)^{2}}{N \Sigma^{l}+\mathcal{K}_{2}^{l}} \\
& =\frac{\left(\mathcal{K}_{1}^{l-1}\right)^{2}}{\gamma_{1}\left(N \Sigma^{l-1}+\mathcal{K}_{2}^{l-1}\right)+\left(\gamma_{1} r^{2}+\gamma_{2}\right)\left(\mathcal{K}_{1}^{l-1}\right)^{2}},
\end{aligned}
$$

where $\gamma_{1}=\overline{K_{i j}^{2}} /{\overline{K_{i i}}}^{2}, \gamma_{2}=\overline{K_{i i}^{2}} /{\overline{K_{i i}}}^{2}$, and $r=q N^{\frac{1}{2}}$. When the superscripts of layer index for $K_{i j}$ and $K_{i i}$ are clear, the superscripts are omitted. Here, we manage to use the activity statistics at previous layers to estimate the dimensionality of the current layer, rather than the original formula [Eq. (12)]. Thus the mechanism for dimensionality change can be revealed.

Note that to evaluate $\gamma_{1}$ and $\gamma_{2}$, we need to compute the following quantities:

$$
\begin{aligned}
& \overline{K_{i i}}=\left\langle\left[\phi^{\prime}\left(x \sqrt{g^{2} Q^{l-1}+\sigma_{b}}\right)\right]^{2}\right\rangle_{x}, \\
& \overline{K_{i i}^{2}}=\left\langle\left[\phi^{\prime}\left(y \sqrt{g^{2} Q^{l-1}+\sigma_{b}}\right)\right]^{4}\right\rangle_{y}, \\
& \overline{K_{i j}^{2}}=\left\langle\left\langle\phi^{\prime}\left(\sqrt{g^{2} \mathcal{K}_{1}^{l-1} x}+\sqrt{g^{2} Q^{l-1}} z_{1}+\sqrt{\sigma_{b}} u_{1}\right)\right\rangle_{x}^{2}\right.
\end{aligned}
$$

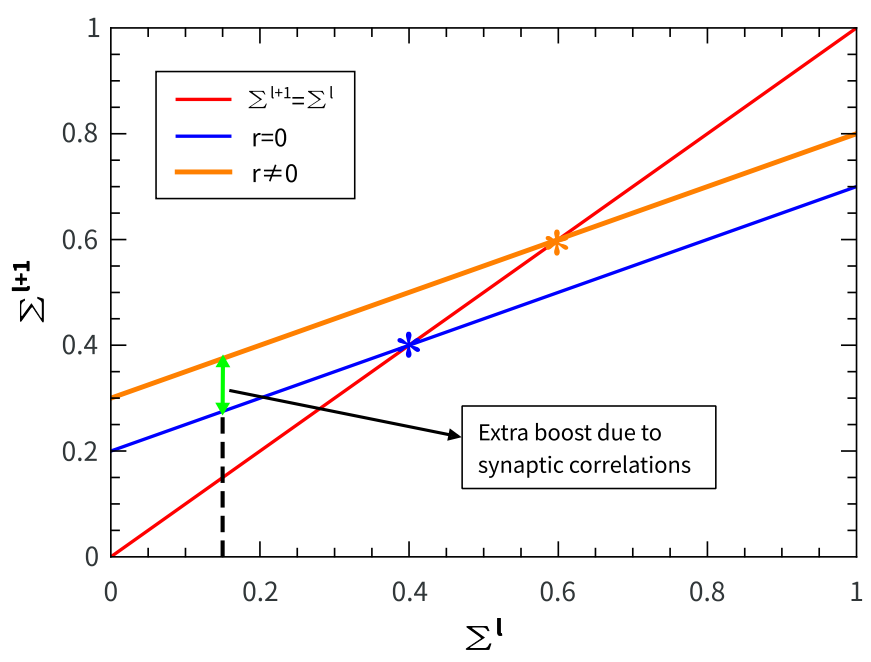

FIG. 2. The schematic illustration showing how synaptic correlations elevate the neural correlation level (multiplied by $N$ ) and the operating point in hidden representations of deep neural networks. The boost is indicated by the double arrow for an example in which the input $\Sigma^{l}$ is below the operating point (indicated by star symbols) where $\Sigma^{l+1}=\Sigma^{l}$. 


$$
\begin{aligned}
& \times\left\langle\phi ^ { \prime } \left[\sqrt{g^{2} \mathcal{K}_{1}^{l-1}} y+\sqrt{g^{2} Q^{l-1}}\left(\rho z_{1}+\sqrt{1-\rho^{2}} z_{2}\right)\right.\right. \\
& \left.\left.\left.+\sqrt{\sigma_{b}} u_{2}\right]\right\rangle_{y}\right\rangle_{z_{1}, z_{2}, u_{1}, u_{2}}
\end{aligned}
$$

where $\rho=q \cdot Q^{l}, \mathcal{K}_{1}^{l}$, and $\mathcal{K}_{2}^{l}$ can also be computed recursively by following the iterative equations in Sec. IIIC1.

\section{Continuous weights}

According to the definition, with the help of Eqs. (35) and (37) and the recursion equation for $\Sigma^{l}$, we obtain

$$
\begin{aligned}
\tilde{D}^{l} & =\frac{\left(\mathcal{K}_{1}^{l}\right)^{2}}{N \Sigma^{l}+\mathcal{K}_{2}^{l}} \\
& =\frac{{\overline{K_{i i}}}^{2} g^{4}\left(\mathcal{K}_{1}^{l-1}\right)^{2}}{\overline{K_{i j}^{2}}\left[g^{4}\left(N \Sigma^{l-1}+\mathcal{K}_{2}^{l-1}\right)+r^{2}\left(\mathcal{K}_{1}^{l-1}\right)^{2}\right]+\overline{K_{i i}^{2}} g^{4}\left(\mathcal{K}_{1}^{l-1}\right)^{2}} \\
& =\frac{\left(\mathcal{K}_{1}^{l-1}\right)^{2}}{\gamma_{1}\left(N \Sigma^{l-1}+\mathcal{K}_{2}^{l-1}\right)+\left(\gamma_{1} \frac{r^{2}}{g^{4}}+\gamma_{2}\right)\left(\mathcal{K}_{1}^{l-1}\right)^{2}},
\end{aligned}
$$

where $\gamma_{1}=\overline{K_{i j}^{2}} /{\overline{K_{i i}}}^{2}, \gamma_{2}=\overline{K_{i i}^{2}} /{\overline{K_{i i}}}^{2}$, and $r=q N^{\frac{3}{2}}$. When the superscripts of layer index for $K_{i j}$ and $K_{i i}$ are clear, the superscripts are omitted. The iterative equations for computing $\gamma_{1}$ and $\gamma_{2}$ are the same with Eq. (47), yet with $\rho=\frac{q N}{g^{2}}$ for the continuous-weight case. $Q^{l}, \mathcal{K}_{1}^{l}$, and $\mathcal{K}_{2}^{l}$ can also be computed recursively by following the iterative equations in Sec. IIIC2.

\section{E. Closed-form mean-field iterations for estimating the dimensionality}

For continuous weights, the mean-field iteration of the covariance matrix of activations is given by

$$
\begin{aligned}
\Delta_{i j}^{l} & =\sum_{k, k^{\prime}} w_{i k}^{l} C_{k k^{\prime}}^{l-1} w_{j k^{\prime}}^{l}, \\
m_{i}^{l} & =\int \operatorname{Dt} \phi\left(\sqrt{\Delta_{i i}^{l}} t+\sum_{j=1}^{N} w_{i j}^{l} m_{j}^{l-1}+b_{i}^{l}\right),
\end{aligned}
$$

and

$$
\begin{aligned}
C_{i j}^{l}= & \int \operatorname{Dx} \operatorname{Dy\phi }\left(\sqrt{\Delta_{i i}^{l}} x+b_{i}^{l}+\sum_{k} w_{i k}^{l} m_{k}^{l-1}\right) \\
& \times \phi\left[\sqrt{\Delta_{j j}^{l}}\left(\psi x+y \sqrt{1-\psi^{2}}\right)+b_{j}^{l}+\sum_{k^{\prime}} w_{j k^{\prime}}^{l} m_{k^{\prime}}^{l-1}\right] \\
& -m_{i}^{l} m_{j}^{l},
\end{aligned}
$$

where $D x=e^{-x^{2} / 2} d x / \sqrt{2 \pi}$, and $\psi=\Delta_{i j}^{l} / \sqrt{\Delta_{i i}^{l} \Delta_{j j}^{l}}$. By taking the covariance matrix $\Lambda$ and zero mean of the data as initial values, we can get the covariance matrix of the activation values of each subsequent layer by iterating the above mean-field equations [Eqs. (49), (50), and (51)]. Then according to the formula $D=(\operatorname{Tr} \mathbf{C})^{2} / \operatorname{Tr} \mathbf{C}^{2}$, the representation dimensionality of each layer can be calculated.

For binary weights, the equation of the mean-field iteration is just a bit different, given by

$$
\begin{aligned}
\Delta_{i j}^{l} & =\frac{g^{2}}{N} \sum_{k, k^{\prime}} w_{i k}^{l} C_{k k^{\prime}}^{l-1} w_{j k^{\prime}}^{l}, \\
m_{i}^{l} & =\int \operatorname{Dt\phi }\left(\sqrt{\Delta_{i i}^{l}} t+\frac{g}{\sqrt{N}} \sum_{j=1}^{N} w_{i j}^{l} m_{j}^{l-1}+b_{i}^{l}\right),
\end{aligned}
$$

and

$$
\begin{aligned}
C_{i j}^{l}= & \int \operatorname{DxDy\phi }\left(\sqrt{\Delta_{i i}^{l}} x+b_{i}^{l}+\frac{g}{\sqrt{N}} \sum_{k} w_{i k}^{l} m_{k}^{l-1}\right) . \\
\phi & {\left[\sqrt{\Delta_{j j}^{l}}\left(\psi x+y \sqrt{1-\psi^{2}}\right)+b_{j}^{l}+\frac{g}{\sqrt{N}} \sum_{k^{\prime}} w_{j k^{\prime}}^{l} m_{k^{\prime}}^{l-1}\right] } \\
& -m_{i}^{l} m_{j}^{l} .
\end{aligned}
$$

The procedure of estimating the linear dimensionality is similar to that of the continuous-weight case.

\section{SUMMARY OF THEORETICAL PREDICTIONS}

Before going to the section of numerical simulations, we summarize the logic of our method and the key theoretical predictions obtained by the lengthy derivations in the previous section. Considering an average over the input ensemble, we write the mean-subtracted weighted sum as $a_{i}^{l}=z_{i}^{l}-\left\langle z_{i}^{l}\right\rangle=$ $\sum_{j} w_{i j}^{l}\left(h_{j}^{l-1}-\left\langle h_{j}^{l-1}\right\rangle\right)$, and thus $a_{i}^{l}$ has zero mean. It follows that the covariance of $\mathbf{a}^{l}$ can be written as $\Delta_{i j}^{l}=\left\langle a_{i}^{l} a_{j}^{l}\right\rangle=$ $\left[\mathbf{w}^{l} \mathbf{C}^{l-1}\left(\mathbf{w}^{l}\right)^{T}\right]_{i j}$, where $\mathbf{C}^{l-1}$ defines the covariance (twopoint correlation) matrix of the hidden representation at the $(l-1)$-th layer. The deep network defined in Fig. 1 implies that each neuron at an intermediate layer receives a large number of nearly independent input terms (weighted activity of previous layers). Therefore, the central limit theorem suggests that the mean of the hidden neural activity $\mathbf{m}^{l}$ and the covariance $\mathbf{C}^{l}$ are given by

$$
\begin{aligned}
& m_{i}^{l}=\left\langle h_{i}^{l}\right\rangle=\int \operatorname{Dt} \phi\left(\sqrt{\Delta_{i i}^{l}} t+\left[\mathbf{w}^{l} \mathbf{m}^{l-1}\right]_{i}+b_{i}^{l}\right) \\
& C_{i j}^{l}=\int \operatorname{DxDy\phi }\left(\sqrt{\Delta_{i i}^{l}} x+b_{i}^{l}+\left[\mathbf{w}^{l} \mathbf{m}^{l-1}\right]_{i}\right) \phi\left[\sqrt{\Delta_{j j}^{l}}\left(\psi x+y \sqrt{1-\psi^{2}}\right)+b_{j}^{l}+\left[\mathbf{w}^{l} \mathbf{m}^{l-1}\right]_{j}\right]-m_{i}^{l} m_{j}^{l},
\end{aligned}
$$

respectively. In Eq. (55), $D x \equiv \frac{e^{-x^{2} / 2} d x}{\sqrt{2 \pi}}$ and $\psi \equiv \frac{\Delta_{i j}^{l}}{\sqrt{\Delta_{i i}^{l} \Delta_{j j}^{l}}}$. To derive Eq. (55), we re-parametrize $a_{i}^{l}$ and $a_{j}^{l}$ by independent normal random variables, such that the covariance structure of the mean-subtracted preactivations is satisfied. In physics, Eq. (55) constructs an iterative mean-field equation across 
layers to describe the transformation of the activity statistics in the deep neural hierarchy.

We then study the linear dimensionality of the hidden representation at each layer, which is given by

$$
D^{l}=\frac{\left(\sum_{i=1}^{N} \lambda_{i}\right)^{2}}{\sum_{i=1}^{N} \lambda_{i}^{2}},
$$

where $\left\{\lambda_{i}\right\}$ is the eigenspectrum of the covariance matrix $\mathbf{C}^{l}$. In statistics, this measure is called the participation ratio [3] that is used to identify the number of nonzero significant eigenvalues. These eigenvalues capture the dominant dimensions that explain variability of the neural representation.

Using the mathematical identities $\operatorname{tr}(\mathbf{C})=\sum_{i} \lambda_{i}$ and $\operatorname{tr}\left(\mathbf{C}^{2}\right)=\sum_{i} \lambda_{i}^{2}$, one can derive that the normalized dimensionality $\tilde{D}^{l} \equiv D^{l} / N=\frac{\left(\mathcal{K}_{1}^{l}\right)^{2}}{N \Sigma^{l}+\mathcal{K}_{2}^{l}}$, where $\Sigma^{l}=\frac{2}{N^{2}} \sum_{i<j}\left(C_{i j}^{l}\right)^{2}$, $\mathcal{K}_{1}^{l} \equiv \frac{1}{N} \sum_{i} C_{i i}^{l}$, and $\mathcal{K}_{2}^{l} \equiv \frac{1}{N} \sum_{i}\left(C_{i i}^{l}\right)^{2} . \Sigma^{l}$ characterizes the overall neural correlation strength. In a mean-field approximation [26], $C_{i j}^{l} \sim \mathcal{O}(1 / \sqrt{N})$, which implies that $\Delta_{i j}^{l}$ is of the same order $\mathcal{O}(1 / \sqrt{N})$. Therefore, $C_{i j}^{l}$ can be expanded in terms of $\Delta_{i j}^{l}$, resulting in $C_{i j}^{l}=K_{i j}^{l} \Delta_{i j}^{l}$ to leading order. Here $K_{i j}^{l} \stackrel{\text { def }}{=} \int D x \phi^{\prime}\left(g \sqrt{\mathcal{K}_{1}^{l-1}} x+z_{i}^{0}\right) \int D y \phi^{\prime}\left(g \sqrt{\mathcal{K}_{1}^{l-1}} y+z_{j}^{0}\right)$ where the mean $z_{i, j}^{0}=b_{i, j}^{l}+\left[\mathbf{w}^{l} \mathbf{m}^{l-1}\right]_{i, j}$.

For the binary-weight case, the preactivation should be multiplied by a prefactor $\frac{g}{\sqrt{N}}$ to ensure that the preactivation is of the order one. By using the above expansion, one gets the relationship between $\Sigma^{l+1}$ and $\Sigma^{l}$ as

$$
N \Sigma^{l+1}=g^{4} \kappa\left[\left(1+q^{2}\right) N \Sigma^{l}+\left(1-q^{2}\right) \mathcal{K}_{2}^{l}+q^{2} N\left(\mathcal{K}_{1}^{l}\right)^{2}\right],
$$

where the overline in $\kappa \equiv \overline{\left(K_{i j}^{l+1}\right)^{2}}$ means the disorder average over the quenched network parameters. It is clear that $q$ cannot be of the order one, in that $N \Sigma \sim \mathcal{O}(1)$ because of the magnitude of $C_{i j}$. A unique scaling for $q$ must then be $q=\frac{r}{\sqrt{N}}$, resulting in $q^{2} N=r^{2}$ where $r \sim \mathcal{O}(1)$, and thus Eq. (57) is self-consistent in physics. We thus call this type of synapses the weakly correlated synapses. Inserting $\Sigma^{l+1}$ into the dimensionality definition together with the linear approximation of $C_{i i}^{l+1} \simeq K_{i i}^{l+1} \Delta_{i i}^{l+1}=g^{2} K_{i i}^{l+1} \mathcal{K}_{1}^{l}$, one immediately obtains the dimensionality of the hidden representation at the $(l+1)$-th layer, in terms of the activity statistics from the previous layer,

$$
\tilde{D}^{l+1}=\frac{\left(\mathcal{K}_{1}^{l}\right)^{2}}{\gamma_{1}\left(N \Sigma^{l}+\mathcal{K}_{2}^{l}\right)+\left(\gamma_{1} r^{2}+\gamma_{2}\right)\left(\mathcal{K}_{1}^{l}\right)^{2}},
$$

where $\gamma_{1} \equiv \frac{\overline{\left(K_{i j}^{l+1}\right)^{2}}}{\overline{K_{i i}^{l+1}}}$, and $\gamma_{2} \equiv \frac{\overline{\left(K_{i i}^{l+1}\right)^{2}}}{{\overline{K_{i i}^{l}}}^{l+1}{ }^{2}}$. Using the CauchySchwartz inequality, one can prove that $\gamma_{1} \leqslant \gamma_{2}$ (see Appendix C). It is also clear that $\gamma_{2} \geqslant 1$.

For the continuous-weight case, following the same line of derivation as above, one obtains a similar relationship between $\Sigma^{l+1}$ and $\Sigma^{l}$ as

$$
N \Sigma^{l+1}=\kappa\left[\left(g^{4}+N^{2} q^{2}\right)\left(N \Sigma^{l}+\mathcal{K}_{2}^{l}\right)+q^{2} N^{3}\left(\mathcal{K}_{1}^{l}\right)^{2}\right] .
$$

The self-consistency of the above equation for the neural correlation strength requires that $q=\frac{r}{N^{3 / 2}}$ where $r \sim \mathcal{O}(1)$. Therefore, $q^{2} N^{2}$ vanishes in a large network width limit. It then follows that the output dimensionality given the input activity statistics reads

$$
\tilde{D}^{l+1}=\frac{\left(\mathcal{K}_{1}^{l}\right)^{2}}{\gamma_{1}\left(N \Sigma^{l}+\mathcal{K}_{2}^{l}\right)+\left(\gamma_{1} r^{2} / g^{4}+\gamma_{2}\right)\left(\mathcal{K}_{1}^{l}\right)^{2}} .
$$

When $q=0, \gamma_{1}=1$, and the dimensionality formula for the orthogonal case is recovered [7]. Note that the dimensionality of the previous hidden representation for both binary- and continuous-weight cases is given by $\tilde{D}^{l}=\frac{\left(\mathcal{K}_{1}^{l}\right)^{2}}{N \Sigma^{l}+\mathcal{K}_{l}^{l}}$. Therefore, the output dimensionality is tuned by a multiplicative factor $\gamma_{1}$ and an additive term [the last term in the denominator of Eq. (58) or Eq. (60)]. The tuning mechanism of the hiddenrepresentation dimensionality in a deep hierarchy is thus much richer than that in the orthogonal scenario [7].

The linear relationship between $\Sigma^{l+1}$ and $\Sigma^{l}$ in Eq. (57) and Eq. (59) defines an operating point where $\Sigma^{l+1}=\Sigma^{l}$. When the input neural correlation strength is below the point, the nonlinear transformation would further strengthen the correlation; whereas the output neural correlation would be attenuated once the input one is above the operating point. The synaptic correlation increases not only the operating point, but also the overall neural correlation level, as indicated by a boost in the intercept while maintaining the slope of the linear relationship in the orthogonal case (Fig. 2).

In the large-width (mean-field) limit, key parameters $\gamma_{1}$, $\gamma_{2}, \mathcal{K}_{1}^{l}$, and $\mathcal{K}_{2}^{l}$ can be iteratively constructed from their values at the input layer (see the previous section). This iteration determines the mean-field solution of the dimension reduction, which captures typical properties of the system under different realizations of the network parameters (quenched disorder).

\section{COMPARISON BETWEEN THEORY AND NUMERICAL SIMULATIONS}

We first study the typical behavior of dimension reduction in networks of binary weights (for numerical details, please see Appendix D). Surprisingly, the weak correlation among synapses is able to reduce further the hidden-representation dimensionality across layers compared to the case of orthogonal weights [Fig. 3(a)]. Moreover, the synaptic correlation $r$ can also boost the correlation strength $\Sigma$ [Fig. 3(b)]. The boost is larger at earlier layers of deep networks. In a practical learning process, synaptic plasticity can introduce a certain level of correlations among synapses, reflecting feature correlations. Our theoretical model predicts that the synaptic correlations can impact both representation manifold and neural decorrelation process. In other words, the weak synaptic correlation enhances the dimension reduction, while reducing the decay speed (with respect to the depth) of the neural correlation strength.

The exact mechanism underlying the computational roles of synaptic correlation can be revealed by a large- $N$ expansion of the two-point correlation function. In the thermodynamic limit, our theory predicts that the dimensionality can be tuned by two factors: one is multiplicative, captured by $\gamma_{1}$, which is observed to grow until arriving at the unity [Fig. 3(d)], and always equal to the unity only at $q=0$. This multiplicative factor [see Eq. (58) or Eq. (60)] gives rise to further 

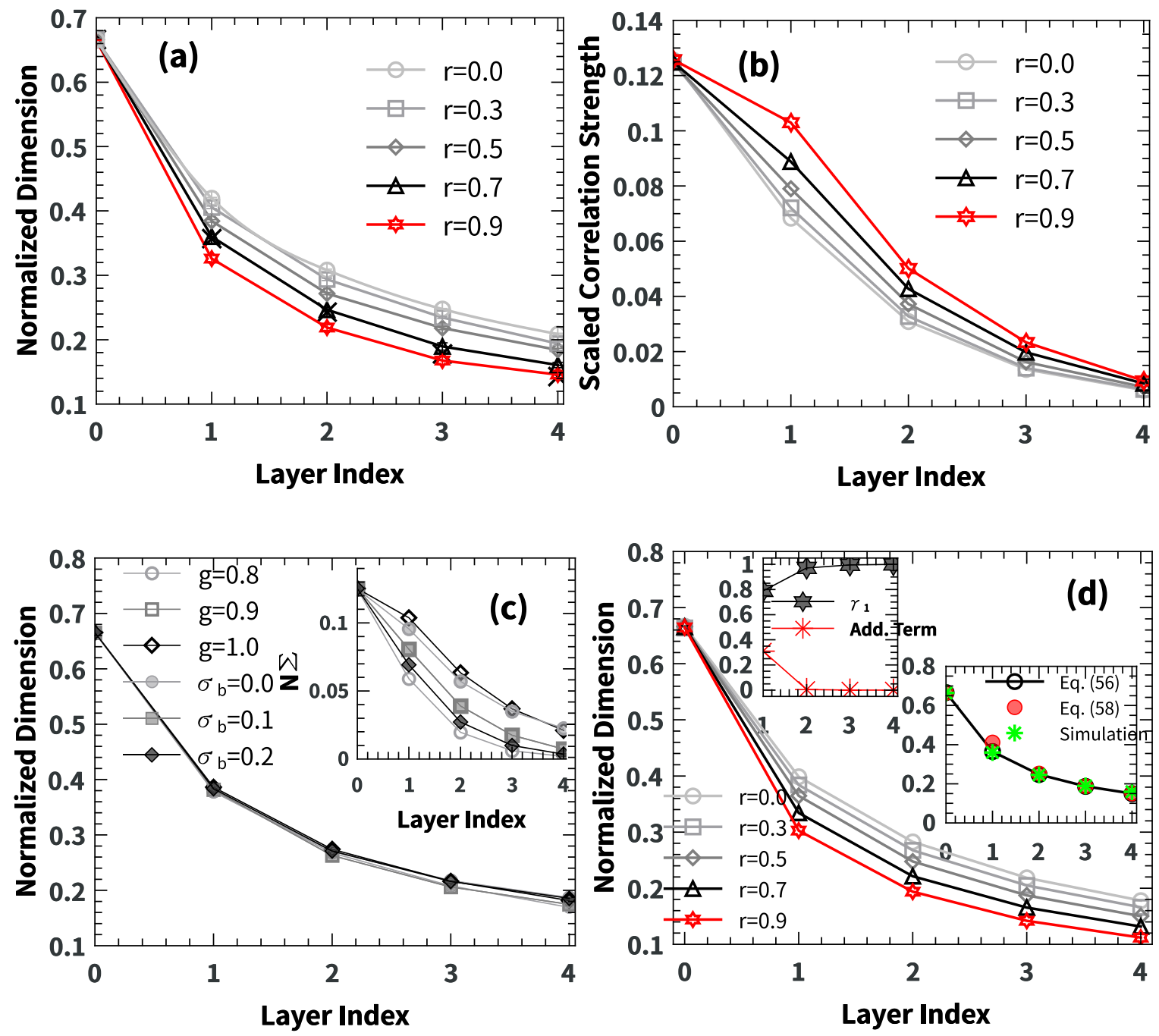

FIG. 3. Typical behavior of dimension reduction in networks of binary weights. Simulations were carried out on networks of finite size $N=200$, and averaged over 10 instances with negligible error bars. (a) Layerwise dimension reduction with different correlation level $r$. $g=0.9, \alpha=2$, and $\sigma_{b}=0.1$. The covariance is obtained by Eq. (55). The cross symbol indicates the simulation result obtained by layerwise propagating $10^{5}$ samples. (b) Layerwise decorrelation with $r$. Other parameters are the same as in (a). The neural correlation strength has been scaled by $N$. (c) Dimension reduction and decorrelation with different values of $g$ and $\sigma_{b} . r=0.5$. $g=0.9$ when $\sigma_{b}$ varies, and $\sigma_{b}=0.1$ when $g$ varies. (d) Large- $N$ limit behavior for $g=0.4$. The left inset shows the behavior of $\gamma_{1}$ and the additive term. The right inset shows a comparison of estimated dimensions between theory and simulation $(N=200)$. In both insets, $r=0.5, \sigma_{b}=0.1$, and $\alpha=2$.

reduction of dimensionality, especially at deeper layers. However, its value can be less than one at earlier layers, thereby allowing possibility of increasing the dimensionality at the $(l+1)$-th layer, provided that $N \Sigma^{l}>\frac{\mathcal{A}}{1-\gamma_{1}}-\mathcal{K}_{2}^{l}$, where $\mathcal{A}>$ 0 denotes the additive term in Eq. (58) or Eq. (60). This predicts that transient dimensionality expansion is possible given strong neural correlations or redundant coding at earlier layers, which was empirically observed during training in both feed-forward and recurrent neural networks [5,7]. The other additive factor is directly related to $r^{2}$. This extra term is clearly positive, thereby contributing an additional reduction of dimensionality. Both factors compete with each other; the multiplicative factor saturates at the unity [the left inset of Fig. 3(d)], while the additive term decreases with the network depth, which overall makes the extent of dimension reduction weaker at deeper layers, in consistent with a practical learning of image classification tasks where the final low-dimensional manifold must be stable for reading out object identities [15,27-31].

The elegant way in which the overall neural correlation level is tuned can also be explained by our theory. The weak synaptic correlation contributes an additional additive term in the linear relationship between the input and output neural correlations. This additive term, namely $r^{2}\left(\mathcal{K}_{1}^{l}\right)^{2}$ [the same for both binary and continuous weights, see Eq. (57) or Eq. (59)], increases the intercept of the linear relationship while maintaining the same slope with the case of $r=0$ (Fig. 2). The decorrelation process would thus proceed less sharply when going deeper into the network. The prediction of our theory may then explain the empirical success in improving the classification performance by a deccorrelation regularization of weights [32]. 


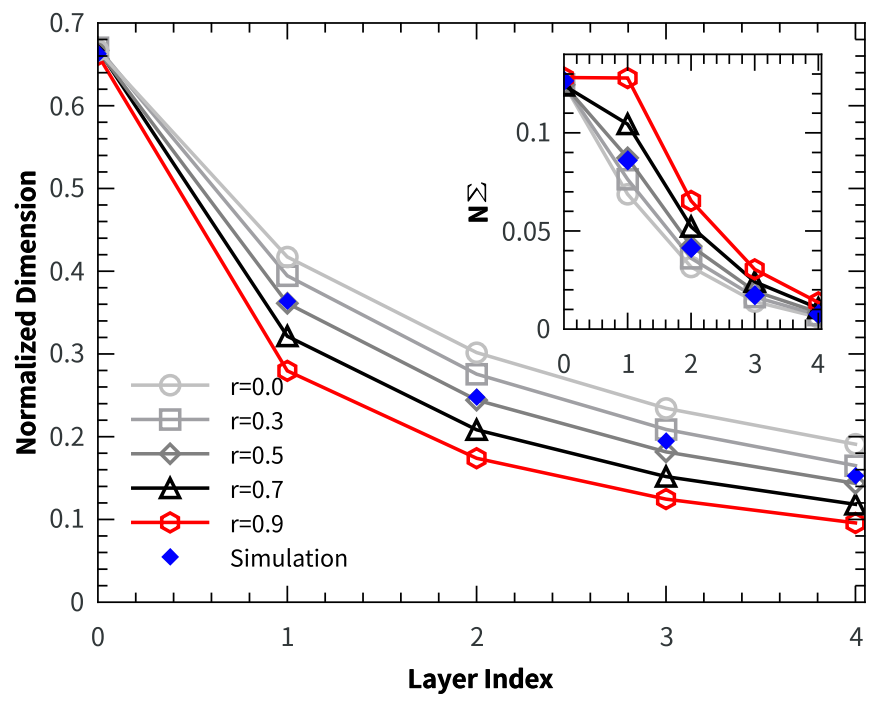

FIG. 4. Typical behavior of dimension reduction in networks of continuous weights using propagation equation Eq. (55). The results are averaged over 10 networks of finite size $N=200$. The error bars are negligible. Simulations indicate layerwise propagating $10^{5}$ samples. $g=0.9, \sigma_{b}=0.1$, and $\alpha=2$.

By changing the other statistics of network parameters, say $g$ and $\sigma_{b}$, but fixing $r$, one can observe rich effects of these parameters [Fig. 3(c)]. First, the dimension reduction seems to be unaffected, or changes by a negligible margin. However, the correlation strength of the hidden representation displays rich behavior. The weight strength elevates the correlation level, as also expected from previous studies [7], playing the similar role to the synaptic correlation [Fig. 3(b)]. In contrast, increasing the firing bias would further decorrelate the hidden representation. The mechanisms are encoded into Eq. (57) and Eq. (59), in that both the weight strength and firing bias affect the slope and intercept of the linear relationship in a highly nontrivial way, via a recursive iteration from initial values of network activity statistics. These rich effects of tuning the neural correlation of hidden representations may thus shed light on understanding the empirical success of reducing overfitting by optimizing decorrelated representations [33]. The decorrelated representation also coincides with the efficient coding hypothesis in system neuroscience [34]. In this hypothesis, a useful representation should be maximally disentangled, rather than being highly redundant. Our analysis provides a theoretical support for this hypothesis, emphasizing the role of weakly correlated synapses. We finally remark that the above analysis also carries over to networks with continuous weights (Fig. 4).

\section{DEEP NEURAL NETWORKS TRAINED WITH HEBBIAN LEARNING RULES}

Our simulations on deep networks trained with Hebbian learning rules $[35,36]$ also show key features of the theoretical predictions of our simple model. To verify the revealed principles in this paper, we perform an on-line training of a layered neural network using the same transfer function, by applying the well-known Hebbian rule. We use the synthetic dataset generated in Sec. A, containing 10000 input samples $(\alpha=2)$. These data samples are then sequentially shown to the input layer of the deep neural network, and then learned layer by layer. We use the synaptic rescaling to control the synaptic strength, like $w_{i j}(t) \leftarrow \frac{g w_{i j}(t)}{\sqrt{\sum_{k} w_{i k}^{2}(t)}}$, where $w_{i j}(t)$ is updated by the following regularized Hebbian rule:

$$
w_{i j}^{l}(t)=w_{i j}^{l}(t-1)+\eta\left[h_{i}^{l} h_{j}^{l-1}-\kappa_{c}\left(\sum_{i^{\prime}: i^{\prime} \neq i} w_{i^{\prime} j}^{l}\right)\left(\sum_{k \neq k^{\prime}}^{N} w_{k j}^{l} w_{k^{\prime} j}^{l}-\sqrt{N} r\right)\right],
$$

where $t$ denotes the learning step, $N$ denotes the receptive field size of the hidden neuron at the $l$ th layer, $\eta$ is the learning rate, and $\kappa_{c}$ enforces the synaptic-correlation constraint, inspired by our theory. The last term in Eq. (61) can be derived as the gradient descent of the correlation-constraint objective:

$$
\Phi_{c}(\mathbf{w})=\frac{\kappa_{c}}{2} \sum_{j}\left[\sum_{i \neq i^{\prime}}^{N} w_{i j} w_{i^{\prime} j}-\sqrt{N} r\right]^{2},
$$

where the synaptic-correlation scaling derived in our paper is used for learning the continuous weights. The synaptic rescaling operation together with the synaptic-correlation constraint encourages synapses to compete with each other to encode input features during learning [35,36].

As shown in Fig. 5, the regularized Hebbian learning rule is able to reduce the dimensionality of hidden representations, while decorrelating the representations as well. The qualitative behavior of the on-line trained systems coincides with the theoretical predictions of our model about roles of synaptic correlations. Therefore, it is promising to design unsupervised or supervised learning algorithms that can control the synaptic correlations in future works, e.g., in sensory perception of real-world datasets.

\section{SUMMARY}

Benefits of weakly correlated synapses are widely claimed in both system neuroscience [4,9-11] and machine learning [32]. The benefits are also realized in a theoretical model of shallow-network unsupervised learning [12], a fundamental process in cerebral cortex [37]. However, the theoretical basis of how weakly correlated synapses promote the computation in deep neural networks remains unexplored, due to the theoretical complexity of handling the covariance matrix of neural responses. This work tackles the challenge, by deriving a mean-field theory for diagonally block-organized correlation matrix of synapses, inspired by our recent work of unsupervised learning [12]. 


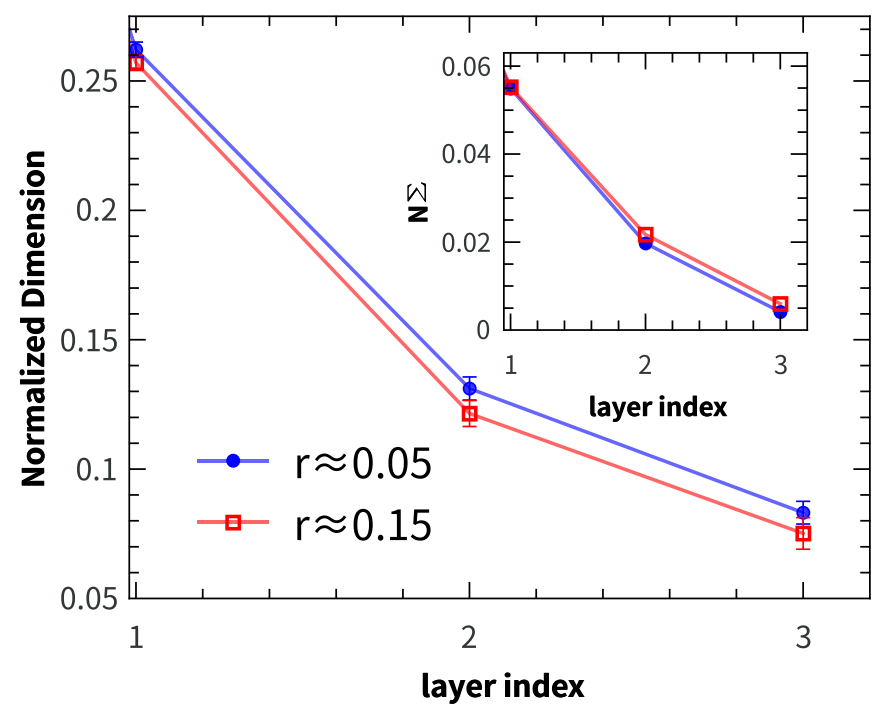

FIG. 5. Dimension reduction and decorrelation in deep Hebbian learning with $N=100 ; \eta=0.0001, \kappa_{c}=0.5, g=0.5$, and 10000 training examples $(\alpha=2)$ are sequentially (on line) shown to the input layer of deep networks. The result is averaged over 10 random realizations.

Our work first identifies the unique scaling of synaptic correlation level, for both binary and continuous weight values, based solely on mathematical self-consistency. This scaling may be experimentally testable, like in the intact brain [38]. The theory then reveals in what exact way the weak synaptic correlation tunes both dimensionality reduction and the associated decorrelation process. More precisely, the synaptic correlation enhances the dimension reduction, while attenuating the decorrelation process. Both computational roles can be explained in a large- $N$ expansion of our mean-field equations. These predictions coincide with empirical successes in decorrelation regularizations of either synaptic level [32] or neural level [33]. Our theory is thus promising to encourage further understanding of how synaptic and neural correlations interact with each other to yield a disentangled representation supporting the success of deep learning, and even hierarchical information processing in different pathways of neural circuits, a challenging problem in interdisciplinary fields across physics, machine learning and neuroscience $[39,40]$.

\section{ACKNOWLEDGMENTS}

We thank Chan Li and Wenxuan Zou for a careful reading of the manuscript. This research was supported by the National Natural Science Foundation of China for Grant No. 11805284 and the start-up budget 74130-18831109 of the 100-talent-program of Sun Yat-sen University.

\section{APPENDIX A: NUMERICAL GENERATION OF INPUT DATA SAMPLES FOLLOWING THE PREDEFINED COVARIANCE}

The input dataset for our deep transformation include 100000 data samples, which are independently sampled from a multivariate Gaussian distribution with zero means and the covariance matrix specified by $\Lambda=\frac{1}{N} \xi \xi^{T}$, where $\xi \in$ $\mathbb{R}^{N \times P}$ and its elements follow a Gaussian distribution $\xi_{i j} \sim$ $\mathcal{N}\left(0, \sigma^{2}\right)$. We define $\alpha=P / N$, and the relation between $\alpha$ and $\tilde{D}$ can be proved to be $\tilde{D}=\alpha /(1+\alpha)$, as we shall show later.

First, we need to calculate the eigenvalue spectrum of $\Lambda$, namely $\left\{\lambda_{i}^{\Lambda}\right\}$ by replica trick [41]. The Edwards-Jones formula reads

$$
\begin{aligned}
\rho(\lambda) & =\frac{1}{N} \sum_{i=1}^{N} \delta\left(\lambda-\lambda_{i}^{\Lambda}\right) \\
& =\frac{1}{N \pi} \lim _{\epsilon \rightarrow 0^{+}} \operatorname{Im} \frac{\partial}{\partial \lambda}\left\langle\ln \operatorname{det}\left(\lambda_{\epsilon} \mathbb{I}-\Lambda\right)\right\rangle_{\Lambda} \\
& =\frac{-2}{\pi N} \lim _{\epsilon \rightarrow 0^{+}} \operatorname{Im} \frac{\partial}{\partial \lambda}\langle\ln Z(\lambda)\rangle_{\Lambda},
\end{aligned}
$$

where the Sokhotski-Plemelj identity is used to derive the second equality, and a Gaussian integral representation of the determinant leads to

$$
Z(\lambda)=\int_{\mathbb{R}^{N}} d \boldsymbol{y} \exp \left[-\frac{1}{2} \boldsymbol{y}^{T}\left(\lambda_{\epsilon} \mathbb{I}-\Lambda\right) \boldsymbol{y}\right],
$$

where $\lambda_{\epsilon}=\lambda-\mathrm{i} \epsilon$, and $d \boldsymbol{y} \stackrel{\text { def }}{=} \prod_{i} \frac{d y_{i}}{\sqrt{2 \pi}}$.

Hereafter, for simplicity, we consider only the annealed average $\langle Z(\lambda)\rangle_{\Lambda}$, instead of the more complex quenched one like that analyzed in Ref. [41]. The result will be cross-checked by numerical experiments. In some cases, the annealed average agrees with the quenched one via replica trick, due to the fact that different replicas of the Gaussian variables $\boldsymbol{y}$ are decoupled [42]. It then proceeds as follows:

$$
\begin{aligned}
\langle Z(\lambda)\rangle & =\int d \boldsymbol{y}\left\langle\exp \left[-\frac{1}{2} \boldsymbol{y}^{T}\left(\lambda_{\epsilon} \mathbb{I}-\Lambda\right) \boldsymbol{y}\right]\right\rangle \\
& =\int d \boldsymbol{y} \exp \left[-\frac{1}{2} \lambda_{\epsilon} \boldsymbol{y}^{T} \boldsymbol{y}\right]\left\langle\exp \left[\frac{1}{2 N} \boldsymbol{y}^{T} \boldsymbol{\xi} \boldsymbol{\xi}^{T} \boldsymbol{y}\right]\right\rangle \\
& =\int d \boldsymbol{y} \exp \left[-\frac{1}{2} \lambda_{\epsilon} \sum_{i=1}^{N} y_{i}^{2}\right]\left\langle\exp \left[\frac{1}{2 N} \sum_{j=1}^{P}\left(\sum_{i=1}^{N} y_{i} \xi_{i j}\right)^{2}\right]\right\rangle
\end{aligned}
$$




$$
\begin{aligned}
& =\int d \boldsymbol{y} \exp \left[-\frac{1}{2} \lambda_{\epsilon} \sum_{i=1}^{N} y_{i}^{2}\right]\left\langle\prod_{j=1}^{P} \exp \left[\frac{1}{2 N}\left(\sum_{i=1}^{N} y_{i} \xi_{i j}\right)^{2}\right]\right\rangle \\
& \propto \int d \boldsymbol{y} d \boldsymbol{m} \exp \left[-\frac{1}{2} \lambda_{\epsilon} \sum_{i=1}^{N} y_{i}^{2}-\sum_{j=1}^{P} m_{j}^{2}\right]\left\langle\prod_{j=1}^{P} \prod_{i=1}^{N} \exp \left[2 \sqrt{\frac{1}{2 N}} m_{j} y_{i} \xi_{i j}\right]\right\rangle,
\end{aligned}
$$

where $d \boldsymbol{m} \stackrel{\text { def }}{=} \prod_{i} \frac{d m_{i}}{\sqrt{\pi}}$, and we have used the integral identity for $\boldsymbol{m}$ :

$$
e^{b^{2}}=\frac{1}{\sqrt{\pi}} \int e^{-x^{2}+2 b x} d x
$$

We then calculate the expectation with respect to $\xi$, and obtain

$$
\begin{aligned}
\left\langle\prod_{j=1}^{P} \prod_{i=1}^{N} \exp \left[2 \sqrt{\frac{1}{2 N}} m_{j} y_{i} \xi_{i j}\right]\right\rangle_{\xi} & =\prod_{j=1}^{P} \prod_{i=1}^{N}\left\langle\exp \left[2 \sqrt{\frac{1}{2 N}} m_{j} y_{i} \xi_{i j}\right]\right\rangle_{\xi_{i j}} \\
& =\prod_{j=1}^{P} \prod_{i=1}^{N} \exp \left[\frac{1}{N} \sigma^{2} m_{j}^{2} y_{i}^{2}\right] .
\end{aligned}
$$

Thus,

$$
\begin{aligned}
\langle Z(\lambda)\rangle \propto & \int d \boldsymbol{y} d \boldsymbol{m} \exp \left[-\frac{1}{2} \lambda_{\epsilon} \sum_{i=1}^{N} y_{i}^{2}-\sum_{j=1}^{P} m_{j}^{2}+\frac{1}{N} \sigma^{2} \sum_{j=1}^{P} m_{j}^{2} \sum_{i=1}^{N} y_{i}^{2}\right] \\
= & \int d \boldsymbol{y} d \boldsymbol{m} d q d r \exp \left[-\frac{1}{2} \lambda_{\epsilon} N q-P r+P \sigma^{2} r q\right] \\
& \times \delta\left(N q-\sum_{i=1}^{N} y_{i}^{2}\right) \delta\left(\operatorname{Pr}-\sum_{j=1}^{P} m_{j}^{2}\right) \\
\propto & \int d \boldsymbol{y} d \boldsymbol{m} d q d r d \hat{q} d \hat{r} \exp \left[-\frac{1}{2} \lambda_{\epsilon} N q-P r+P \sigma^{2} r q\right] \\
& \times \exp \left[N q \hat{q}-\hat{q} \sum_{i=1}^{N} y_{i}^{2}\right] \exp \left[\operatorname{Pr} \hat{r}-\hat{r} \sum_{j=1}^{P} m_{j}^{2}\right] \\
\propto & \int d q d r d \hat{q} d \hat{r} \exp \left[-\frac{1}{2} \lambda_{\epsilon} N q-\operatorname{Pr}+P \sigma^{2} r q\right] \\
& \times \exp \left[N q \hat{q}-\frac{N}{2} \ln \hat{q}\right] \exp \left[\operatorname{Pr} \hat{r}-\frac{P}{2} \ln \hat{r}\right],
\end{aligned}
$$

where we have used the following identity:

$$
\int d y e^{-a y^{2}}=\sqrt{\frac{\pi}{a}}=\sqrt{\pi} e^{-\frac{1}{2} \ln a} .
$$

We can now write down $\langle Z(\lambda)\rangle$ as

$$
\langle Z(\lambda)\rangle=\int d q d r d \hat{q} d \hat{r} \exp \left[-N f_{\lambda}(q, r, \hat{q}, \hat{r})\right],
$$

where

$$
\begin{aligned}
f_{\lambda}(q, r, \hat{q}, \hat{r})= & \frac{1}{2} \lambda_{\epsilon} q+\alpha r-\alpha \sigma^{2} r q-q \hat{q}+\frac{1}{2} \ln \hat{q} \\
& -\alpha r \hat{r}+\frac{\alpha}{2} \ln \hat{r} .
\end{aligned}
$$

When $N \rightarrow \infty$, we can use Laplace's approximation, from which

$$
\langle Z(\lambda)\rangle \simeq \exp \left[-N f_{\lambda}\left(q^{\star}, r^{\star}, \hat{q}^{\star}, \hat{r}^{\star}\right)\right] .
$$

The stationary point $\left(q^{\star}, r^{\star}, \hat{q}^{\star}, \hat{r}^{\star}\right)$ is computed as

$$
\begin{aligned}
& \frac{\partial f_{\lambda}}{\partial q}=0 \Rightarrow \frac{1}{2} \lambda_{\epsilon}-\alpha \sigma^{2} r-\hat{q}=0, \\
& \frac{\partial f_{\lambda}}{\partial r}=0 \Rightarrow \alpha-\alpha \sigma^{2} q-\alpha \hat{r}=0, \\
& \frac{\partial f_{\lambda}}{\partial \hat{q}}=0 \Rightarrow q-\frac{1}{2 \hat{q}}=0, \\
& \frac{\partial f_{\lambda}}{\partial \hat{r}}=0 \Rightarrow \alpha r-\frac{\alpha}{2 \hat{r}}=0 .
\end{aligned}
$$




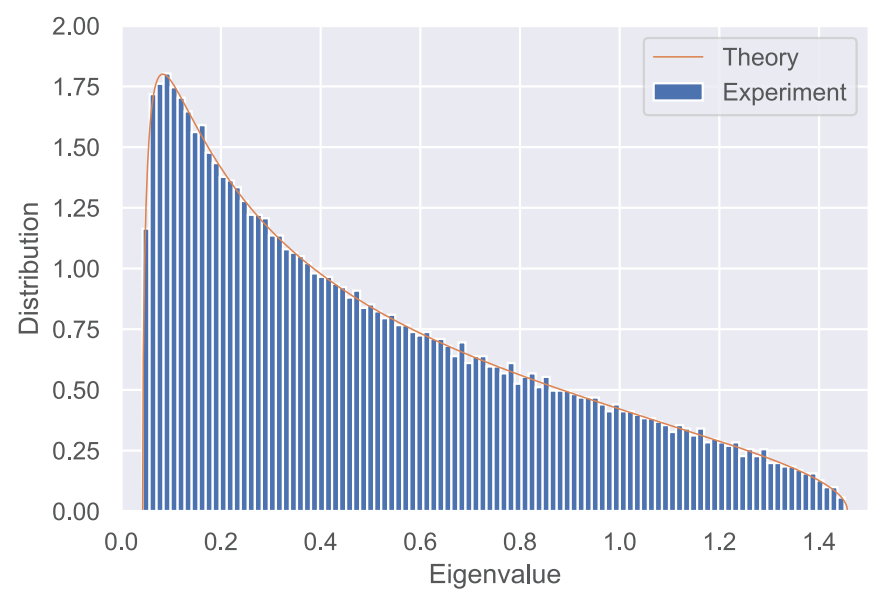

FIG. 6. The eigenvalue distribution of the covariance matrix $\Lambda$. Here we set $\alpha=2, \sigma=0.5$ and $N=5000$.

Applying the Edwards-Jones formula in the annealed version, we obtain

$$
\begin{aligned}
\rho(\lambda) & =\frac{-2}{\pi N} \lim _{\varepsilon \rightarrow 0^{+}} \operatorname{Im} \frac{\partial}{\partial \lambda} \ln \langle Z(\lambda)\rangle \\
& \simeq \frac{-2}{\pi N} \lim _{\varepsilon \rightarrow 0^{+}} \operatorname{Im} \frac{\partial}{\partial \lambda}\left[-N f_{\lambda}\left(q^{\star}, r^{\star}, \hat{q}^{\star}, \hat{r}^{\star}\right)\right] .
\end{aligned}
$$

Note that $\frac{\partial f_{\lambda}}{\partial \lambda}=\frac{1}{2} q^{\star}$, where $q^{\star}$ can be obtained by solving the saddle-point equations [Eq. (A11)]. We finally arrive at

$$
\rho(\lambda) \simeq \frac{1}{\pi} \lim _{\epsilon \rightarrow 0^{+}} \operatorname{Im}\left[q^{\star}\right]=\frac{\sqrt{\left(\sigma^{2} \lambda_{+}-\lambda\right)\left(\lambda-\sigma^{2} \lambda_{-}\right)}}{2 \pi \sigma^{2} \lambda},
$$

where $\lambda_{ \pm}=(\sqrt{\alpha} \pm 1)^{2}$ for $\alpha>1$. The comparison between theory and simulation is shown in Fig. 6.

In the large- $N$ limit, the normalized dimensionality of the matrix $\Lambda$ can be written in the form as follows:

$$
\tilde{D}=\frac{\left(\sum_{i} \lambda_{i}\right)^{2}}{N \sum_{i} \lambda_{i}^{2}}=\frac{\left(\frac{1}{N} \sum_{i} \lambda_{i}\right)^{2}}{\frac{1}{N} \sum_{i} \lambda_{i}^{2}}=\frac{\left(\int d \lambda \rho(\lambda) \lambda\right)^{2}}{\int d \lambda \rho(\lambda) \lambda^{2}} .
$$

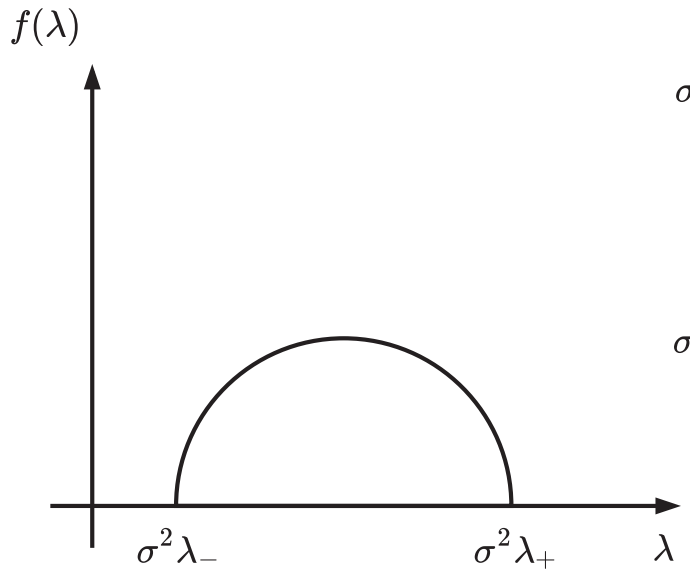

(a)
The numerator part is given by the first-order moment of the eigenspectrum,

$$
\begin{aligned}
I_{1} & \stackrel{\text { def }}{=} \int d \lambda \rho(\lambda) \lambda \\
& =\frac{1}{2 \pi \sigma^{2}} \int_{\lambda_{-}}^{\lambda_{+}} \sqrt{\left(\lambda-\sigma^{2} \lambda_{-}\right)\left(\sigma^{2} \lambda_{+}-\lambda\right)} d \lambda \\
& =\frac{1}{2 \pi \sigma^{2}} \frac{1}{2} \pi\left[\frac{\sigma^{2}\left(\lambda_{+}-\lambda_{-}\right)}{2}\right]^{2} \\
& =\alpha \sigma^{2},
\end{aligned}
$$

where we calculate the integral by computing the area of the semicircle shown in Fig. 7(a). The denominator part is given by the second moment of the spectrum, computed as

$$
\begin{aligned}
I_{2} & \stackrel{\text { def }}{=} \int d \lambda \rho(\lambda) \lambda^{2} \\
& =\frac{1}{2 \pi \sigma^{2}} \int_{\lambda_{-}}^{\lambda_{+}} \lambda \sqrt{\left(\lambda-\sigma^{2} \lambda_{-}\right)\left(\sigma^{2} \lambda_{+}-\lambda\right)} d \lambda \\
& =\frac{1}{2 \pi \sigma^{2}} \frac{1}{2} \pi\left[\frac{\sigma^{2}\left(\lambda_{+}-\lambda_{-}\right)}{2}\right]^{2} \frac{\sigma^{2}\left(\lambda_{+}+\lambda_{-}\right)}{2} \\
& =\sigma^{4} \alpha(\alpha+1),
\end{aligned}
$$

where the integral here can be transformed to half of the volume of the cylinder shown in Fig. 7(b) [43]. Finally, we conclude that

$$
\tilde{D}=\frac{I_{1}^{2}}{I_{2}}=\frac{\alpha}{\alpha+1},
$$

from which the normalized input dimensionality is independent of the input-pattern $(\xi)$ variance $\sigma^{2}$. This analytic result is confirmed in numerical simulations in the main text.

\section{APPENDIX B: DIMENSION CORRELATES OF SUPERVISED AND UNSUPERVISED LEARNING}

In this section, we show numerical evidences of dimension correlates of the learning performances in both unsupervised and supervised learning. We first show how dimensionality

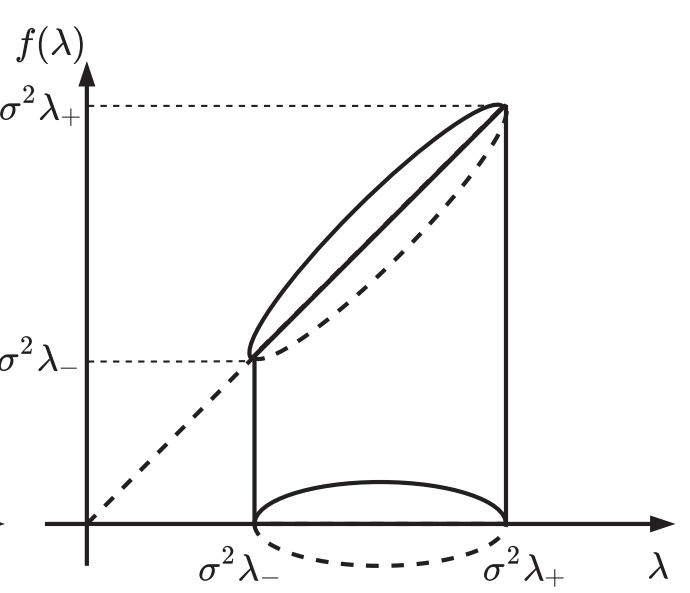

(b)

FIG. 7. The calculation of spectrum moments by a geometric method. $f(\lambda)$ denotes the integrand of $I_{1}$ (a) or $I_{2}$ (b). 


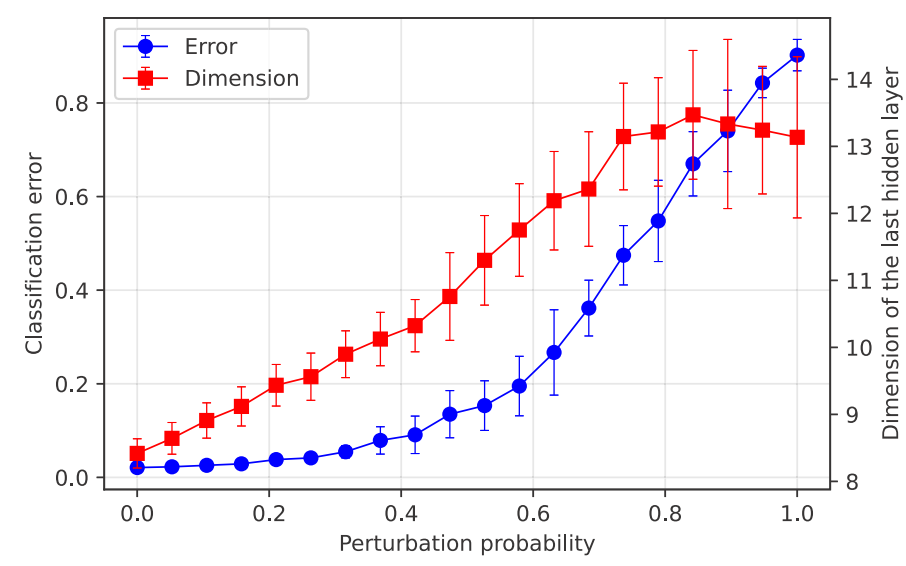

(a)

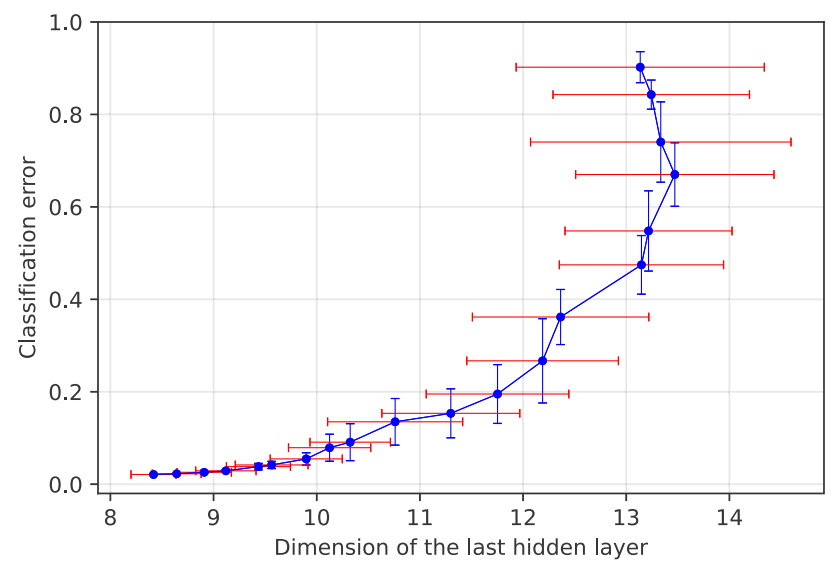

(b)

FIG. 8. A four-layered MLP is trained with backpropagation. The full training dataset of MNIST is used with a batch size of 512. The number of training epochs is set to 120 . The initial learning rate is 0.001 , which is multiplied by 0.5 every 20 epochs. The dimension is estimated by layerwise propagating the training dataset. (a) The dimension of the last hidden layer and the classification error versus perturbation probability. (b) The classification error versus the dimension of the last hidden layer under different perturbation strength. The error bars indicate standard deviations across 20 independent experiments, i.e., 20 MLPs are trained from independent initializations.

of the last hidden layer in a multilayered perceptron (MLP) correlates with the classification performance. The input data is given by the MNIST dataset with 10 classes of handwritten digits [44]. The MLP is trained by the standard backpropagation algorithm [45], which adjusts each network weight to minimize the cross-entropy loss between the actual output and the target one-hot output. The trained MLP has two hidden layers of width equal to 100 neurons. After training, each connection between the two hidden layers is perturbed with a probability $p$. The perturbation is implemented by replacing the trained weight value by a Gaussian random variable subject to $\mathcal{N}(0,1 / N)$ where $N=100$ (width of hidden layers). We found that the test performance (classification error rate on the unseen handwritten digits) correlates with the dimensionality of the last hidden layer (Fig. 8). In particular, when $p$ gets large, the activity manifold in the last hidden layer is strongly modified by the perturbed input connectivity. In other words, the dimensionality increases as the performance deteriorates. Therefore, a proper (low) dimensionality must be maintained for a good performance of a deep network, like MLP here. This important relationship between low-dimensional compressed representations and generalization properties of deep networks was also observed in previous works studying more complex settings [30,31].

For the unsupervised learning, we apply the contrast divergence algorithm to train a deep belief network (DBN), which is composed of multiple restricted Boltzmann machines (RBMs) [46]. The DBN is fed with the handwritten digits dataset (the MNIST dataset). The network parameters (weights and biases) are updated by the contrast divergence algorithm to maximize the log-likelihood of the real data. The training proceeds in a layerwise manner. The number of input pixels is equal to 784 , and then RBMs of hidden layer size $N=200$ are stacked on top of each other to learn features in the input data in an unsupervised way (i.e., no labels needed). The reconstruction error $\epsilon \equiv\left\|\mathbf{v}-\mathbf{v}^{\prime}\right\|_{2}^{2}$ measures the learning performance. $\mathbf{v}$ is the input, while $\mathbf{v}^{\prime}$ is the reconstructed one after one contrast divergence step. The reconstruction error can be transformed to the average percentage of pixels the reconstructed input does not match the original input, which we call $f_{\text {err }}$.

We observed that for different reconstruction errors, the dimensionality of the hidden layers can either decrease across layers or have a transient expansion at earlier layers followed by a reduction (Fig. 9). This shows evidences that the dimensionality of hidden representations plays a vital role in unsupervised learning as well. In particular, the reconstruction error correlates with the rich behavior of dimension reduction. Figure 10 displays how the dimensionality and correlation strength of hidden activities evolve during training (the first 60 epochs as an example). These two key quantities reach steady values at the end of training.

Next, we show typical examples of weight statistics in the unsupervised learning experiments. To extract an entire ensemble of weights is computationally challenging. In practice, we train three DBNs independently and obtain three sets of weights. We then choose three typical neurons (say, $i, j, k)$ from the visible layer, and extract their weights connected to the next layer of size $N$. We obtain a vector $\left[w_{1 i}, w_{2 i}, \ldots, w_{N i} ; w_{1 j}, \ldots, w_{N j} ; w_{1 k}, \ldots, w_{N k}\right]$ of the size $3 N$, and each of them can be organized into a matrix form (we call it the forward weight matrix, whose elements' correlation is modeled in our work). Figure 11 shows the numerical results of the weight statistics, which confirms that the weight fluctuation can emerge from a practical training with a diverse structure. Our main assumption of weight statistics is a simplified setting of a practical training, making a theoretical study (hard but essential) tractable.

To sum up, we show dimension correlates of learning performances in both supervised and unsupervised learning in this section. In addition, a significant weight fluctuation (i.e., depending on independent experiments) is also observed in a practical training. Inspired by these observations, our model setting in the main text disentangles exactly key 


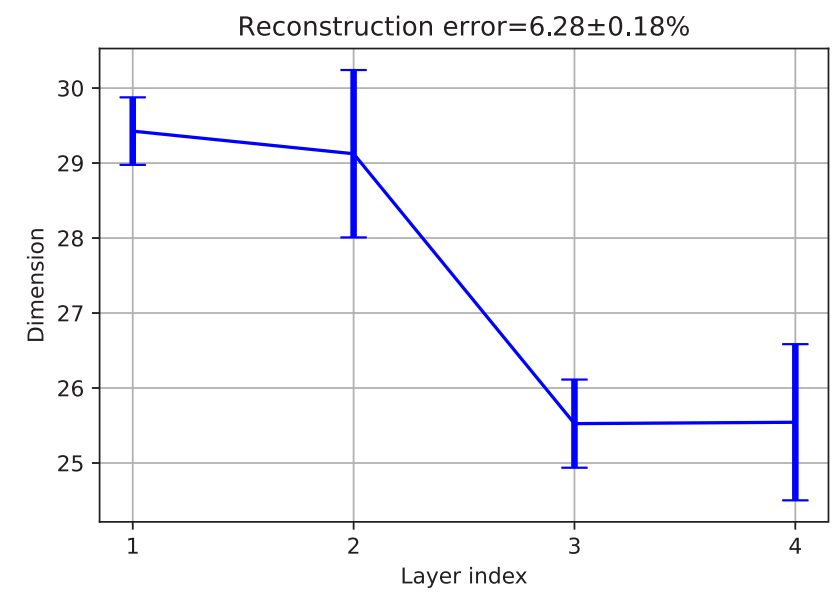

(a)

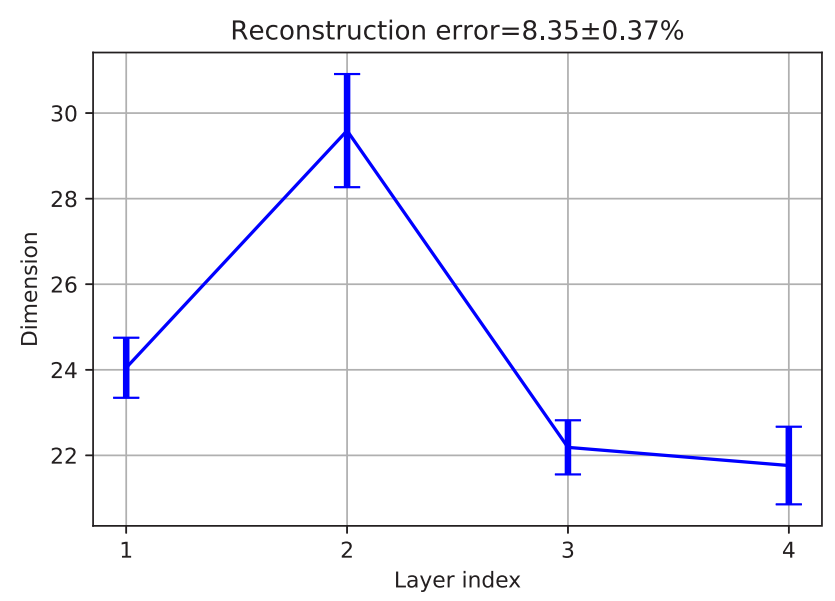

(b)

FIG. 9. The linear dimension of each hidden layer versus hidden layer index. A DBN with four hidden layers is trained. Each hidden layer has 200 neurons. The hidden neural activity takes a binary value $( \pm 1)$. The training dataset consists of 10000 MNIST digits. The hidden layer of each stacked RBM uses binary activities $( \pm 1)$ during training. During training next RBM, the activities of the visible layer of that RBM are the average activities of the hidden layer in the lower-level RBM, and thus are real values ranged from -1 to 1 . We estimate the reconstruction error of the entire DBN through Gibbs sampling which assumes that all activities are binary values $( \pm 1)$. We set the batch size to be 100 . The initial weights are independently sampled from the Gaussian distribution $\mathcal{N}(0,1 / N)$, where $N$ is the width of the previous layer. The initial biases are independently sampled from the standard Gaussian distribution $\mathcal{N}(0,1)$. To accelerate the training process, we use the Nesterov's momentum. The value of the momentum parameter starts with 0.5 , and after the reduction of the reconstruction error becomes slow, this value is changed to be 0.9 . We also use the L2 weight decay with the strength $10^{-4}$. The dimension is estimated by layerwise propagating the training dataset. (a) The DBN has a typical reconstruction error of $f_{\text {err }}=6.28 \pm 0.18 \%$. (b) The DBN has a typical reconstruction error of $f_{\text {err }}=8.35 \pm 0.37 \%$. The error bar is estimated from 20 independent trainings.

factors affecting dimension reduction in a correlated-synapses scenario, thereby providing a first step toward understanding these complex yet intriguing phenomena of dimension correlates.

\section{APPENDIX C: PROOF OF $\gamma_{1} \leqslant \gamma_{2}$}

In this section, we prove the relation $\gamma_{1} \leqslant \gamma_{2}$ at an arbitrary layer $l$, where $\gamma_{1}=\overline{K_{i j}^{2}} /{\overline{K_{i i}}}^{2}$ and $\gamma_{2}={\overline{K_{i i}^{2}}}_{/ K_{i i}}^{2}$. Because the denominators of $\gamma_{1}$ and $\gamma_{2}$ are the same, we just need to prove that $\overline{K_{i j}^{2}} \leqslant \overline{K_{i i}^{2}}$, where

$$
\begin{aligned}
\overline{K_{i i}^{2}}= & \left\langle\left[\phi^{\prime}\left(\sqrt{g^{2} \mathcal{K}_{1}^{l-1}} t+x \sqrt{g^{2} Q^{l-1}+\sigma_{b}}\right)\right]^{4}\right\rangle_{x, t} \\
\overline{K_{i j}^{2}}= & \left\langle\left\langle\phi^{\prime}\left(\sqrt{g^{2} \mathcal{K}_{1}^{l-1}} x+\sqrt{g^{2} Q^{l-1}} z_{1}+\sqrt{\sigma_{b}} u_{1}\right)\right\rangle_{x}^{2}\right. \\
& \times\left\langle\phi ^ { \prime } \left[\sqrt{g^{2} \mathcal{K}_{1}^{l-1}} y+\sqrt{g^{2} Q^{l-1}}\left(\rho z_{1}+\sqrt{1-\rho^{2}} z_{2}\right)\right.\right. \\
& \left.\left.\left.+\sqrt{\sigma_{b}} u_{2}\right]\right\rangle_{y}^{2}\right\rangle_{z_{1}, z_{2}, u_{1}, u_{2}}
\end{aligned}
$$

Note that we add higher-order contributions of $\Delta_{i i}^{l}$ back into $\overline{K_{i i}^{2}}$, as mentioned in Sec. III B. To proceed, we define $\vartheta_{x} \stackrel{\text { def }}{=}$ $\sqrt{g^{2} \mathcal{K}_{1}^{l-1}} x+\sqrt{g^{2} Q^{l-1}} z_{1}+\sqrt{\sigma_{b}} u_{1}$ and $\vartheta_{y} \stackrel{\text { def }}{=} \sqrt{g^{2} \mathcal{K}_{1}^{l-1}} y+$ $\sqrt{g^{2} Q^{l-1}}\left(\rho z_{1}+\sqrt{1-\rho^{2}} z_{2}\right)+\sqrt{\sigma_{b}} u_{2}$. Let $\left\langle\phi^{\prime}\left(\vartheta_{x}\right)\right\rangle_{x}^{2}=\Phi_{1}$ and $\left\langle\phi^{\prime}\left(\vartheta_{y}\right)\right\rangle_{y}^{2}=\Phi_{2}$. According to the Cauchy-Schwartz inequality, we have

$$
{\overline{\Phi_{1} \Phi_{2}}}^{2} \leqslant \overline{\Phi_{1}^{2}} \cdot \overline{\Phi_{2}^{2}}
$$

where - denotes the quenched disorder average as before. It is then easy to show that $\overline{\Phi_{2}^{2}}=\overline{\Phi_{1}^{2}}=\overline{K_{i i}^{2}}$. We finally conclude that $\gamma_{1} \leqslant \gamma_{2}$.

\section{APPENDIX D: NUMERICAL GENERATION OF WEIGHT-CORRELATED NEURAL NETWORKS}

We consider a five-layer fully connected neural network with one input layer and four hidden layers. The number of neurons in each layer is specified by $N$. The parameters of the network are generated by following the procedure below, and after the initialization, all parameters remain unchanged during the simulation of dimension estimation, and then the result is averaged over many independent realizations of the same statistics of network parameters.

\section{Binary weights}

The binary weight $\left(w_{i j}= \pm 1\right)$ follows a statistics of zero mean and the covariance specified by

$$
\overline{w_{i j}^{l} w_{k s}^{l}}=\delta_{j s} q+\delta_{i k} \delta_{j s}(1-q)=q \delta_{j s}\left(1-\delta_{i k}\right)+\delta_{i k} \delta_{j s} .
$$

Diagonalization of the full covariance matrix of binary weights is challenging. However, no correlation occurs within each RF. Then we can generate the network weights for each diagonal block in Fig. 1 (see the main text) independently by a 


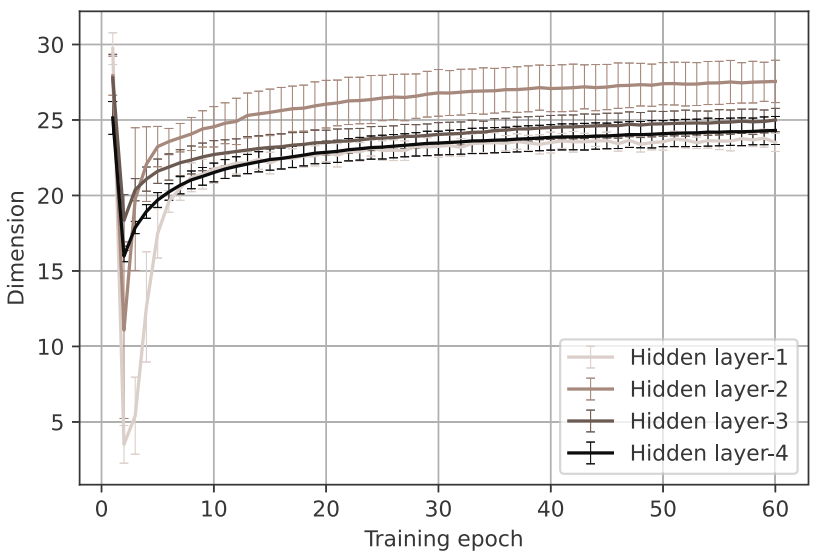

(a)

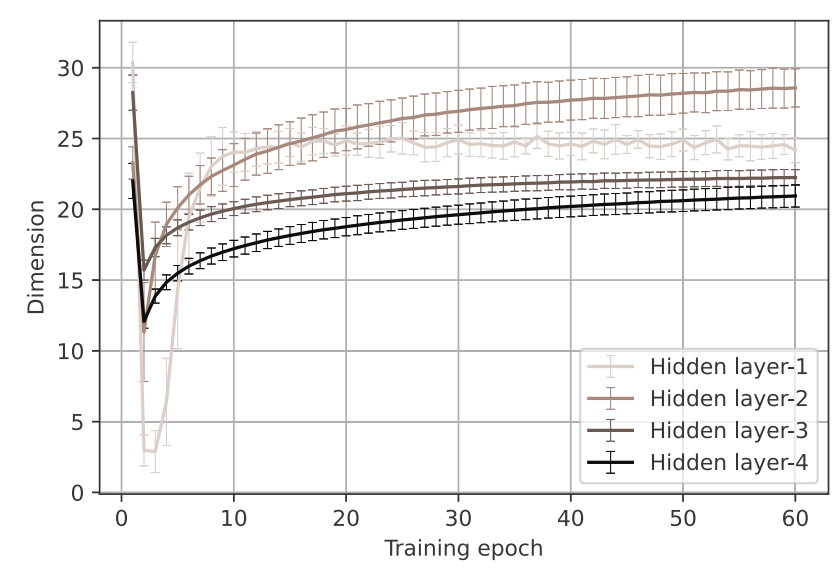

(c)

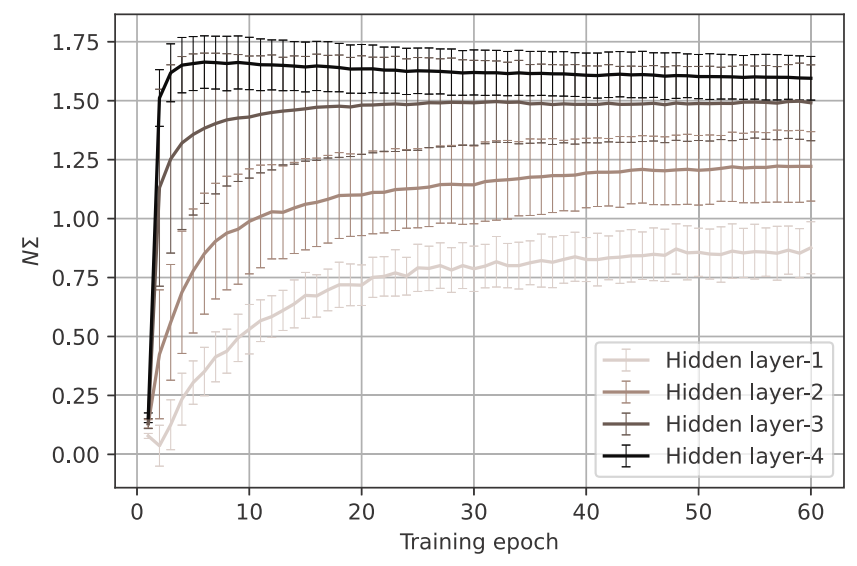

(b)

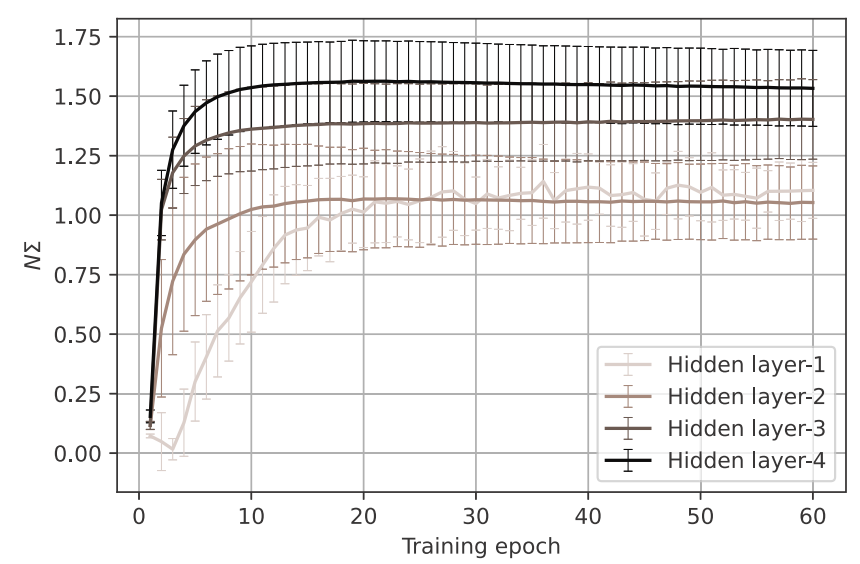

(d)

FIG. 10. The dimension and correlation strength (multiplied by the width of the corresponding layer) during the process of training. The training conditions are the same as in Fig. 9. When calculating the covariance matrix of the activities, we use the real-valued average activities of neurons [7]. The dimension is also estimated by layerwise propagating the training dataset. [(a) and (b)] The DBNs with a typical reconstruction error of $6.28 \pm 0.18 \%$. [(c) and (d)] The DBNs with a typical reconstruction error of $8.35 \pm 0.37 \%$. Here we just show the first 60 epochs. The error bar is estimated from 20 independent trainings.

dichotomized Gaussian (DG) process [47]. In the DG process, the binary weights can be generated by $w_{i j}^{l}=\operatorname{sign}\left(x_{i j}^{l}\right)$, where

$$
\operatorname{sgn}(x)= \begin{cases}1 & x \geqslant 0 \\ -1 & x<0\end{cases}
$$

where $x_{i j}^{l}$ is sampled from a multivariate Gaussian distribution of zero mean (due to $\overline{w_{i j}^{l}}=0$ ) and the following covariance, as also shown in a schematic illustration in Fig. 12,

$$
\overline{x_{i j}^{l} x_{k s}^{l}}=\delta_{j s} \Sigma+\delta_{i k} \delta_{j s}(1-\Sigma)=\Sigma \delta_{j s}\left(1-\delta_{i k}\right)+\delta_{i k} \delta_{j s} .
$$

The relation between $q$ and $\Sigma$ can be established by matching the covariance of the DG process with our prescribed correlation level $q$, i.e.,

$$
q=\iint D x D y \operatorname{sgn}(x) \operatorname{sgn}\left(\Sigma x+\sqrt{1-\Sigma^{2}} y\right)=\frac{2}{\pi} \arcsin \Sigma .
$$

Then, we have

$$
\Sigma=\sin \frac{\pi q}{2} .
$$

A sample of the multivariate Gaussian distribution with the $N \times N$ covariance matrix $\boldsymbol{\Sigma}$ (diagonal blocks in Fig. 12) can be obtained by first carrying out a Cholesky decomposition of the covariance, i.e., $\boldsymbol{\Sigma}=\mathbf{L} \mathbf{L}^{T}$, where $\mathbf{L}$ is a lower-triangular matrix. A sample is then obtained as $\mathbf{z}=\mathbf{L} \boldsymbol{\epsilon}$, where $\boldsymbol{\epsilon} \sim$ $\mathcal{N}(\mathbf{0}, \mathbb{I})$. I denotes an identity matrix. The parameter $b_{i}^{l}$ follows $\mathcal{N}\left(0, \sigma_{b}\right)$ independently.

\section{Continuous weights}

For the continuous case, the parameter $w_{i j}^{l}$ follows the Gaussian distribution with zero means and the covariance specified by

$\overline{w_{i j}^{l} w_{k s}^{l}}=\delta_{j s} q+\delta_{i k} \delta_{j s}\left(\frac{g^{2}}{N}-q\right)=q \delta_{j s}\left(1-\delta_{i k}\right)+\frac{g^{2}}{N} \delta_{i k} \delta_{j s}$.

When generating the weights, we divide $\mathbf{w}^{l}$ into $N$ vectors, each of which is defined by $\left[w_{1 j}^{l}, w_{2 j}^{l}, \ldots, w_{N j}^{l}\right](j=$ $1,2, \ldots, N)$. Then all those vectors are independently sampled from a multivariate Gaussian distribution with zero 


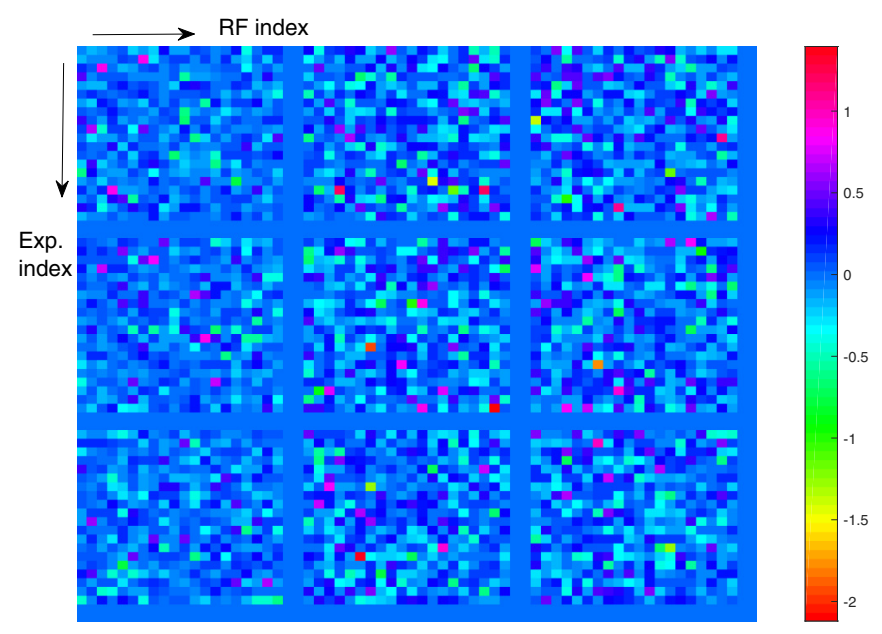

FIG. 11. The forward weight matrix (the first RBM in the DBN as an example) for three typical neurons in the visible layer, under different independent training experiments. Each row represents three forward weight matrices organized in a $20 \times 20$ form. Each column indicates different experimental results of the same visibleneuron's forward weight matrix. The training conditions are the same as in Fig. 9, except for the hidden width of $N=400$.

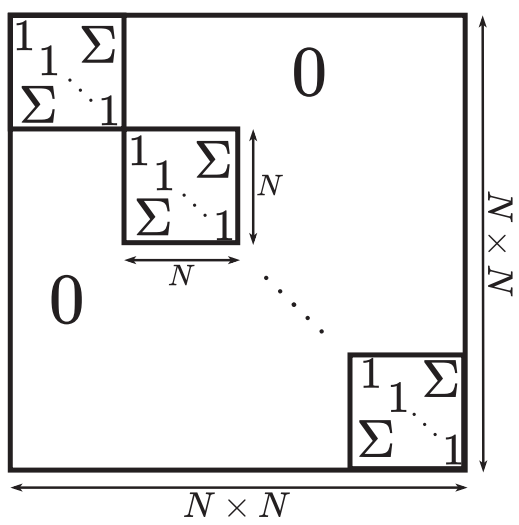

FIG. 12. The schematic illustration of the covariance matrix of $\boldsymbol{x}^{l}$ used to generate correlated binary weights.

means and the covariance matrix $\boldsymbol{\Sigma} \in \mathbb{R}^{N \times N}$, in which $\boldsymbol{\Sigma}$ is a symmetric matrix with diagonal elements $g^{2} / N$ and off-diagonal elements $q$. The parameter $b_{i}^{l}$ follows $\mathcal{N}\left(0, \sigma_{b}\right)$ independently.
[1] M. R. Cohen and A. Kohn, Measuring and interpreting neuronal correlations, Nat. Neurosci. 14, 811 (2011).

[2] F. Franke, M. Fiscella, M. Sevelev, B. Roska, A. Hierlemann, and R. Silveira, Structures of neural correlation and how they favor coding, Neuron 89, 409 (2016).

[3] K. Rajan, L. Abbott, and H. Sompolinsky, Inferring stimulus selectivity from the spatial structure of neural network dynamics, in Advances in Neural Information Processing Systems 23, edited by J. D. Lafferty, C. K. I. Williams, J. Shawe-Taylor, R. S. Zemel, and A. Culotta, (Curran Associates, Red Hook, NY, 2010), pp. 1975-1983.

[4] N. Brunel, Is cortical connectivity optimized for storing information?, Nat. Neurosci. 19, 749 (2016).

[5] M. Farrell, S. Recanatesi, T. Moore, G. Lajoie, and E. SheaBrown, Recurrent neural networks learn robust representations by dynamically balancing compression and expansion, bioRxiv (2019), doi: 10.1101/564476.

[6] N. Alex Cayco-Gajic and R. Angus Silver, Re-evaluating circuit mechanisms underlying pattern separation, Neuron 101, 584 (2019).

[7] H. Huang, Mechanisms of dimensionality reduction and decorrelation in deep neural networks, Phys. Rev. E 98, 062313 (2018).

[8] G. E. Hinton and R. R. Salakhutdinov, Reducing the dimensionality of data with neural networks, Science 313, 504 (2006).

[9] K. Harris and T. Mrsic-Flogel, Cortical connectivity and sensory coding, Nature 503, 51 (2013).

[10] K. Harris and G. Shepherd, The neocortical circuit: Themes and variations, Nat. Neurosci. 18, 170 (2015).

[11] A. A. Koulakov, T. Hromádka and A. M. Zador, Correlated connectivity and the distribution of firing rates in the neocortex, J. Neurosci. 29, 3685 (2009).
[12] T. Hou, K. Y. Michael Wong, and H. Huang, Minimal model of permutation symmetry in unsupervised learning, J. Phys. A: Math. Theor. 52, 414001 (2019).

[13] T. Hou and H. Huang, Statistical Physics of Unsupervised Learning with Prior Knowledge in Neural Networks, Phys. Rev. Lett. 124, 248302 (2020).

[14] A. Achille and S. Soatto, Emergence of invariance and disentanglement in deep representations, J. Mach. Learn. Res. 19, 1 (2018).

[15] M. Pagan, L. S. Urban, M. P. Wohl, and N. C. Rust, Signals in inferotemporal and perirhinal cortex suggest an untangling of visual target information, Nat. Neurosci. 16, 1132 (2013).

[16] J. J. DiCarlo and D. D. Cox, Untangling invariant object recognition, Trends Cognit. Sci. 11, 333 (2007).

[17] KiJung Yoon, M. A. Buice, C. Barry, R. Hayman, N. Burgess, and I. R. Fiete, Specific evidence of low-dimensional continuous attractor dynamics in grid cells, Nat. Neurosci. 16, 1077 (2013).

[18] R. C. Williamson, B. R. Cowley, A. Litwin-Kumar, B. Doiron, A. Kohn, M. A. Smith, and B. M. Yu, Scaling properties of dimensionality reduction for neural populations and network models, PLOS Comput. Biol. 12, e1005141 (2016).

[19] J. A. Gallego, M. G. Perich, L. E. Miller, and S. A. Solla, Neural manifolds for the control of movement, Neuron 94, 978 (2017).

[20] E. Warnberg and A. Kumar, Perturbing low dimensional activity manifolds in spiking neuronal networks, PLOS Comput. Biol. 15, e1007074 (2019).

[21] C. J. Cueva, A. Saez, E. Marcos, A. Genovesio, M. Jazayeri, R. Romo, C. Daniel Salzman, M. N. Shadlen, and S. Fusi, Lowdimensional dynamics for working memory and time encoding, Proc. Natl. Acad. Sci. USA 117, 23021 (2020). 
[22] D. L. K. Yamins and J. J. DiCarlo, Using goal-driven deep learning models to understand sensory cortex, Nat. Neurosci. 19, 356 (2016).

[23] S. S. Schoenholz, J. Gilmer, S. Ganguli, and J. Sohl-Dickstein, Deep information propagation, arXiv:1611.01232 (2016).

[24] B. Hanin and D. Rolnick, How to start training: The effect of initialization and architecture, arXiv:1803.01719 (2018).

[25] J. Lee, Y. Bahri, R. Novak, S. S. Schoenholz, J. Pennington, and J. Sohl-Dickstein, Deep neural networks as gaussian processes, arXiv:1711.00165 (2017).

[26] M. Mézard, G. Parisi, and M. A. Virasoro, Spin Glass Theory and Beyond (World Scientific, Singapore, 1987).

[27] C. P. Hung, G. Kreiman, T. Poggio, and J. J. DiCarlo, Fast readout of object identity from macaque inferior temporal cortex, Science 310, 863 (2005).

[28] T. Serre, A. Oliva, and T. Poggio, A feedforward architecture accounts for rapid categorization, Proc. Natl. Acad. Sci. USA 104, 6424 (2007).

[29] H. Hong, D. L. K. Yamins, N. J. Majaj, and J. J. DiCarlo, Explicit information for category-orthogonal object properties increases along the ventral stream, Nat. Neurosci. 19, 613 (2016).

[30] A. Ansuini, A. Laio, J. H. Macke, and D. Zoccolan, Intrinsic dimension of data representations in deep neural networks, arXiv:1905.12784 (2019).

[31] S. Recanatesi, M. Farrell, M. Advani, T. Moore, G. Lajoie, and E. Shea-Brown, Dimensionality compression and expansion in deep neural networks, arXiv:1906.00443 (2019).

[32] P. Rodriguez, J. Gonzalez, G. Cucurull, J. M. Gonfaus, and X. Roca, Regularizing CNNs with locally constrained decorrelations, arXiv:1611.01967 (2016).

[33] M. Cogswell, F. Ahmed, R. Girshick, L. Zitnick, and D. Batra, Reducing overfitting in deep networks by decorrelating representations, arXiv:1511.06068 (2015).

[34] H. Barlow, Possible principles underlying the transformation of sensory messages, in Sensory Communication, edited by W. Rosenblith (MIT Press, Cambridge, MA, 1961), pp. 217-234.
[35] K. D. Miller and D. J. C. MacKay, The role of constraints in hebbian learning, Neural Comput. 6, 100 (1994).

[36] H. Sebastian Seung and J. Zung, A correlation game for unsupervised learning yields computational interpretations of hebbian excitation, anti-hebbian inhibition, and synapse elimination, arXiv:1704.00646 (2017).

[37] D. Marr, A Theory for Cerebral Neocortex, Proc. R. Soc. Lond. B 176, 161 (1970).

[38] J. Barral and A. Reyes, Synaptic scaling rule preserves excitatory-inhibitory balance and salient neuronal network dynamics, Nat. Neurosci. 19, 1690 (2016).

[39] A. Saxe, S. Nelli, and C. Summerfield, If deep learning is the answer, then what is the question? Nat. Rev. Neurosci. 22, 55 (2020).

[40] D. Hassabis, D. Kumaran, C. Summerfield, and M. Botvinick, Neuroscience-inspired artificial intelligence, Neuron 95, 245 (2017).

[41] S. F. Edwards and R. C. Jones, The eigenvalue spectrum of a large symmetric random matrix, J. Phys. A: Math. Gen. 9, 1595 (1976).

[42] H. J. Sommers, A. Crisanti, H. Sompolinsky, and Y. Stein, Spectrum of Large Random Asymmetric Matrices, Phys. Rev. Lett. 60, 1895 (1988).

[43] Chen Zi-Ming (private communication, 2020).

[44] Y. Lecun, L. Bottou, Y. Bengio, and P. Haffner, Gradient-based learning applied to document recognition, Proc. IEEE 86, 2278 (1998).

[45] D. E. Rumelhart, G. E. Hinton, and R. J. Williams, Learning representations by back-propagating errors, Nature 323, 533 (1986).

[46] G. Hinton, S. Osindero, and Y. Teh, A fast learning algorithm for deep belief nets, Neural Comput. 18, 1527 (2006).

[47] J. H. Macke, P. Berens, A. S. Ecker, A. S. Tolias, and M. Bethge, Generating spike trains with specified correlation coefficients, Neural Comput. 21, 397 (2009). 